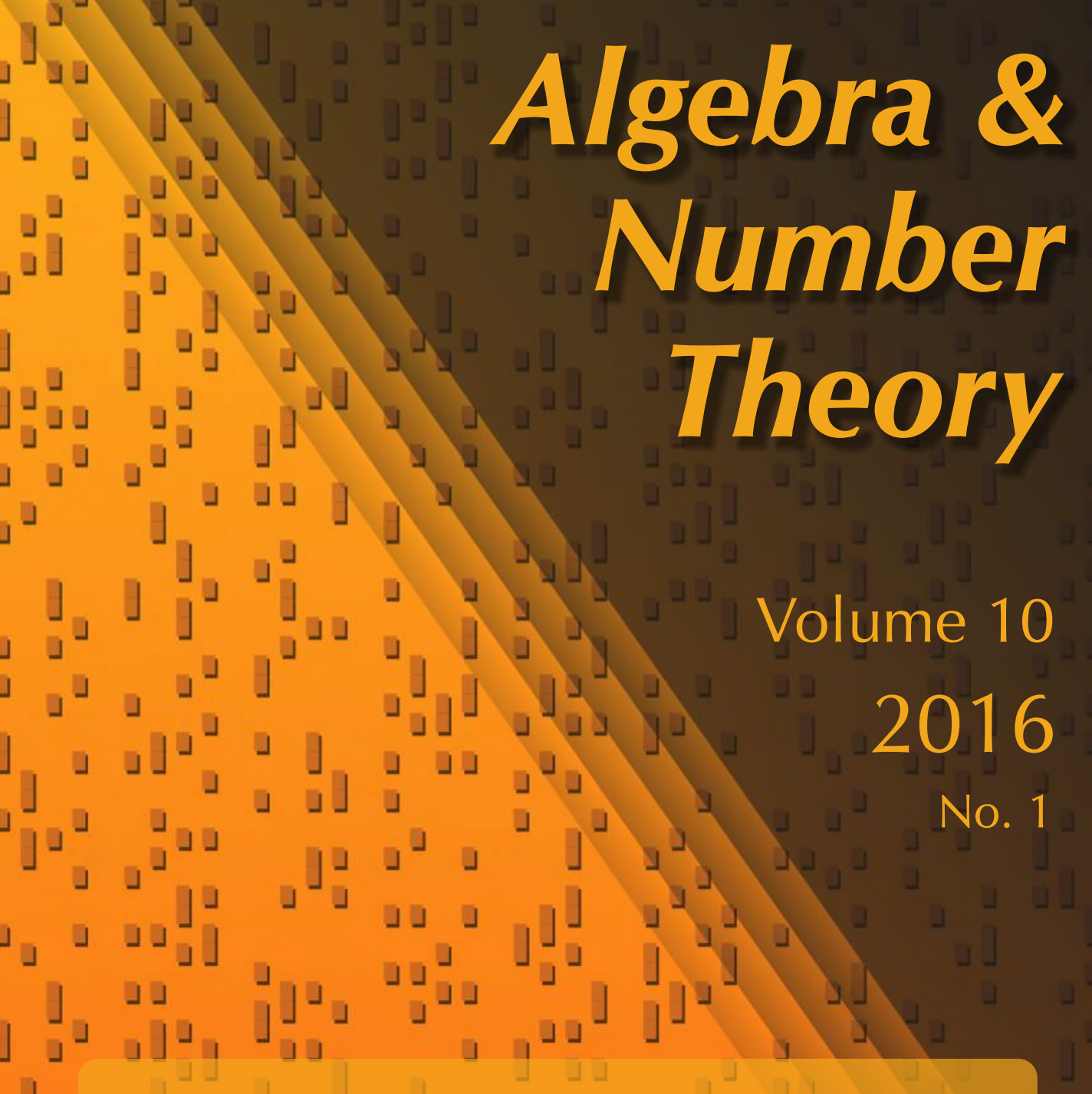

\title{
On tensor factorizations of Hopf algebras
}

$\downarrow$ Marc Keilberg and Peter Schauenburg $\perp$

\lrcorner$\lrcorner$

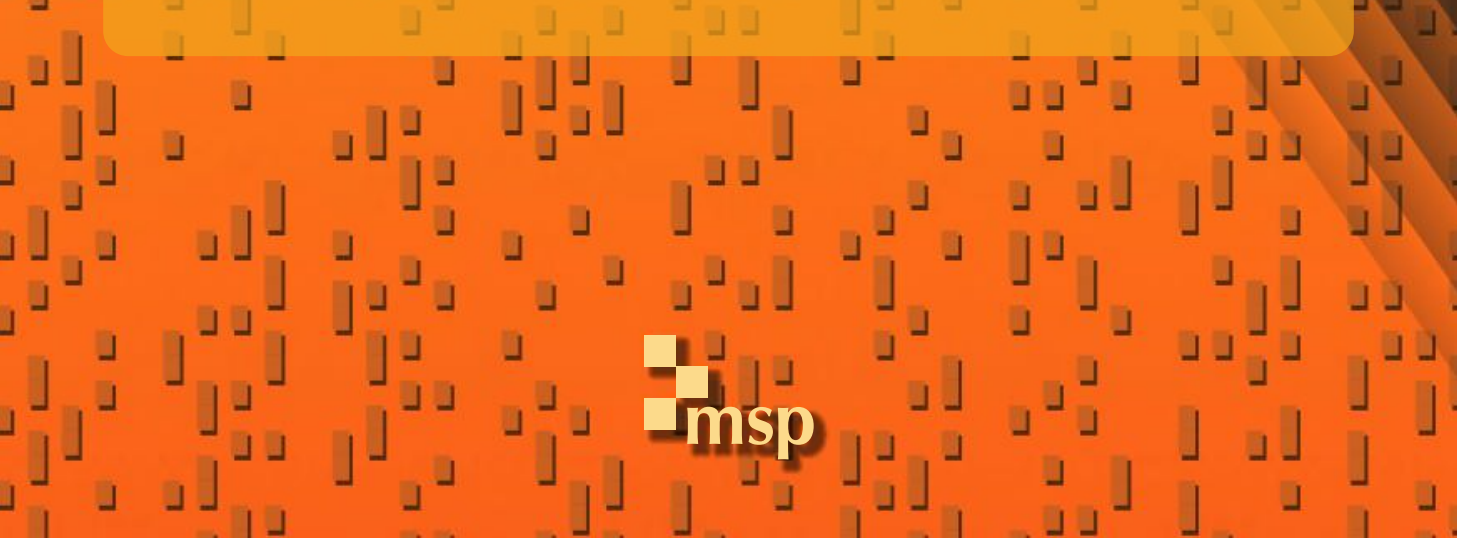




\title{
On tensor factorizations of Hopf algebras
}

\author{
Marc Keilberg and Peter Schauenburg
}

\begin{abstract}
We prove a variety of results on tensor product factorizations of finite dimensional Hopf algebras (more generally Hopf algebras satisfying chain conditions in suitable braided categories). The results are analogs of well-known results on direct product factorizations of finite groups (or groups with chain conditions) such as Fitting's lemma and the uniqueness of the Krull-Remak-Schmidt factorization. We analyze the notion of normal (and conormal) Hopf algebra endomorphisms, and the structure of endomorphisms and automorphisms of tensor products. The results are then applied to compute the automorphism group of the Drinfeld double of a finite group in the case where the group contains an abelian factor. (If it doesn't, the group can be calculated by results of the first author.)
\end{abstract}

\section{Introduction}

The larger part of this paper is concerned with general results on Hopf algebras in braided categories generalizing well-known results from the theory of finite groups (or groups with chain conditions), such as Fitting's lemma, the Krull-RemakSchmidt decomposition, and a description of endomorphisms and automorphisms of products of Hopf algebras. The last section deals with the description of the automorphism group of the Drinfeld double $\mathcal{D}(G)$ of a finite group $G$. This last problem was the starting point of our work.

In the case that $G$ has no nontrivial abelian direct factors, a complete description of the automorphisms was given in [Keilberg 2015]. The case when $G$ has such an abelian factor was left open. We will write such a group as $G=C \times H$, where $H$ has no nontrivial abelian direct factors and $C$ is abelian. In this case we naturally have that $\mathcal{D}(G) \cong \mathcal{D}(C) \otimes \mathcal{D}(H)$ is a tensor product of Hopf algebras.

Thus, we are naturally led to analyze endomorphisms and automorphisms of a tensor product of two Hopf algebras. In [Bidwell et al. 2006; Bidwell 2008] an analysis of the automorphisms of direct products of groups was provided. The basic idea is to describe such automorphisms by a matrix of morphisms between the factors. The machinery of normal group endomorphisms and Fitting's lemma then allows one to

Research partially supported through a FABER Grant by the Conseil régional de Bourgogne. MSC2010: primary 16T05; secondary 18D35, 18D10, 20D99, 81R05.

Keywords: Hopf algebra, factorization, Fitting's lemma, Krull-Remak-Schmidt. 
deduce conditions on the various morphisms from conditions on the factors. For example, when the two factors have no common direct factors, then the diagonal terms of the matrix have to be automorphisms. In Section 8 we derive suitably analogous results for tensor product Hopf algebras. Before this can be done, however, we have to carry over to our Hopf algebraic setting some basic notions and classical results from group theory. In Section 2 we develop the terminology of commuting morphisms (for groups these are just morphisms whose images commute) and dually of cocommuting morphisms, and in Section 3 the notions of normal and conormal Hopf endomorphisms. The analog of Fitting's lemma which will produce tensor product decompositions from binormal endomorphisms and thus, under suitable circumstances, common tensor factors from certain endomorphisms of tensor products, will be proved in Section 5. An important application of Fitting's lemma in group theory is the uniqueness of the Krull-Remak-Schmidt decomposition, which we prove in Section 6. Extensions of the Krull-Remak-Schmidt decomposition were studied previously in [Burciu 2011] for decompositions of semisimple Hopf algebras into simple semisimple tensor factors. By contrast our techniques make no use of semisimplicity but only of chain conditions. It is also worth noting that the Krull-Remak-Schmidt result shows that our results are specific to Hopf algebras and cannot be readily generalized to finite or even fusion tensor categories: Müger [2003] gives an example where the factors in the decomposition of a fusion category into prime factors are not unique.

In fact the above results on the structure theory of finite dimensional Hopf algebras over a field $\mathbb{k}$ will be developed in greater generality for Hopf algebras in braided abelian tensor categories that fulfill chain conditions on Hopf subalgebras and quotient Hopf algebras. Apart from the fact that the results will thus immediately apply to objects like super-Hopf algebras, for some purposes the categorical setting is simply very natural, since it allows treating mutually dual notions like normality and conormality or ascending and descending chain conditions on the same footing. If the braiding of the base category is not a symmetry, then some of our basic objects of study may be hard to come by: it is well known that the tensor product of two Hopf algebras in a braided monoidal category can only be formed if the two factors are "unbraided", that is, if the braiding between them behaves like a symmetry. On the other hand, some of our results imply that tensor product decompositions have to exist in certain situations. Thus these results also imply that the braiding has to be "partially trivial". For example, if nonnilpotent normal endomorphisms of a Hopf algebra exist, they have to be isomorphisms by Fitting's lemma unless the braiding is partially trivial. An automorphism of a tensor product of nonisomorphic Hopf algebras (necessarily "unbraided" between each other) has to induce automorphisms on the factors, unless the braiding is partially trivial on one of the factors.

Section 4 deals with some technical issues raised by our categorical framework. In preparation for Fitting's lemma we decompose a Hopf algebra with chain conditions, 
for which a Hopf algebra endomorphism is given, into a Radford biproduct (in the generalized braided version due to Bespalov and Drabant [1998]). A technical result on (co)invariants under Hopf algebra endomorphisms has some bearing on the notions of epimorphisms and monomorphisms studied notably for infinite dimensional Hopf algebras in [Chirvăsitu 2010].

In Section 9 we present the application of the general results on the structure of finite Hopf algebras and their automorphisms to the study of automorphisms of Drinfeld doubles of groups. Letting $G=C \times H$ as before, taking the field to be the complex numbers, and defining $\widehat{H}$ to be the group of linear characters of $H$, then under the isomorphisms $\mathcal{D}(C) \cong \mathbb{C}(\widehat{C} \times C)$ and $\widehat{C} \times C \cong C^{2}$ the result can be stated as

$$
\operatorname{Aut}(\mathcal{D}(C \times H)) \cong\left(\begin{array}{cc}
\operatorname{Aut}\left(C^{2}\right) & \operatorname{Hom}^{c}\left(\mathcal{D}(H), \mathbb{C} C^{2}\right) \\
\operatorname{Hom}\left(C^{2}, \widehat{H} \times Z(H)\right) & \operatorname{Aut}(\mathcal{D}(H))
\end{array}\right) .
$$

The only term not explicitly determined by [Keilberg 2015] or standard methods for finite abelian groups is $\operatorname{Hom}^{c}\left(\mathcal{D}(H), \mathbb{C} C^{2}\right)$, which we define in Section 8. In this case the morphisms can be described entirely in terms of group homomorphisms and central subgroups of $G$ satisfying certain relations [Agore et al. 2014; Keilberg 2015], so the description is not a significant problem. In Example 9.10 we completely describe $\operatorname{Aut}\left(\mathcal{D}\left(D_{2 n}\right)\right)$ where $D_{2 n}$ is the dihedral group of order $2 n$, for the case $n \equiv 2 \bmod 4$ and $n>2$. This is precisely when there is an isomorphism $D_{2 n} \cong \mathbb{Z}_{2} \times D_{n}$. From this we can easily provide a formula for the order of $\operatorname{Aut}\left(\mathcal{D}\left(D_{2 n}\right)\right)$. In particular we find that $\operatorname{Aut}\left(\mathcal{D}\left(D_{12}\right)\right)$ has order $1152=2^{7} 3^{2}$.

\section{Preliminaries and notation}

Throughout the paper, $\mathcal{B}$ is an abelian braided tensor category with braiding $\tau$; we will assume that $\mathcal{B}$ is strict, backed up by the well-known coherence theorems. Algebras, coalgebras, bialgebras, Hopf algebras are in $\mathcal{B}$. All undecorated Homs, Ends, etc., will be for morphisms of Hopf algebras or groups, as appropriate. We will use the following graphical notations to do computations in $\mathcal{B}$ : the braiding is

$$
\tau_{V W}=\underbrace{V W}_{W V} \text { and } \tau_{V W}^{-1}=\underbrace{W V}_{V W} .
$$

We shall say that the objects $V$ and $W$ are unbraided if $\tau_{V W}=\tau_{W V}^{-1}$.

Multiplication and unit of an algebra $A$, and comultiplication and counit of a coalgebra $C$ are

$$
\nabla_{A}=\underbrace{A}_{A}, \quad \eta_{A}=\underbrace{}_{A}, \quad \Delta_{C}=\overbrace{C}^{C}, \quad \varepsilon_{C}=\stackrel{l}{C} .
$$


The antipode of a Hopf algebra and, if it exists, its inverse are<smiles>O=[IH]</smiles>

In order to have a straightforward notion of Hopf subalgebra and quotient Hopf algebra of a given Hopf algebra, we shall assume that tensor products in $\mathcal{B}$ are exact in each argument.

An object in $\mathcal{B}$ satisfies the ascending chain condition on subobjects if and only if it satisfies the descending chain condition on quotient objects, by which we understand the descending chain condition on subobjects in the opposite category. For Hopf algebras we will use the descending chain conditions on Hopf subalgebras and on quotient Hopf algebras. When a Hopf algebra satisfies the descending chain conditions on both Hopf subalgebras and quotient Hopf algebras, we simply say that it satisfies both chain conditions.

If $f: H \rightarrow G$ is a Hopf algebra morphism, we define the right and left $f$ coinvariant subobjects of $H$ as being the equalizers

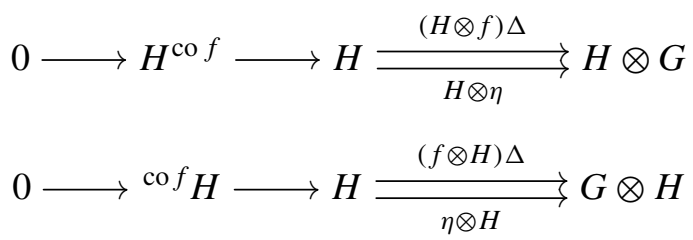

And dually, the left and right invariant quotients by coequalizers

$$
\begin{aligned}
& H \otimes G \underset{\varepsilon \otimes G}{\stackrel{\nabla(f \otimes G)}{\longrightarrow}} G \longrightarrow H \backslash G \longrightarrow 0
\end{aligned}
$$

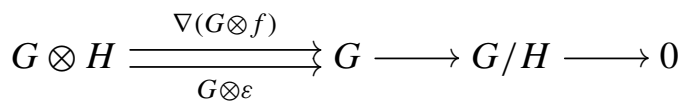

We note that the coinvariant subobjects are subalgebras of $H$, and the invariant quotients are quotient coalgebras of $G$.

We will say a Hopf algebra is abelian if it is both commutative and cocommutative. When working over a field, abelian semisimple Hopf algebras are precisely the duals of abelian group algebras, up to a separable field extension [Montgomery 1993, Theorem 2.3.1]. We will say a Hopf algebra is nonabelian when it is not abelian.

\section{Commuting and cocommuting morphisms}

In this section we formulate an obvious commutation condition for morphisms to an algebra (for ordinary algebras it just means that elements in the respective images 
commute) and its dual, and we collect equally obvious consequences that will be useful in later calculations. We note that for each and every fact on Hopf algebras in a braided category there is a dual fact. We will not always state, but still freely use the duals of our statements

Let $A$ be an algebra, $V, W \in \mathcal{B}$, and $f: V \rightarrow A, g: W \rightarrow A$ morphisms in $\mathcal{B}$. We say that $f$ and $g$ multiplication commute and write $f \curlyvee g$ if $\nabla(g \otimes f)=\nabla(f \otimes g) \tau$ $(=\nabla \tau(g \otimes f))$, or graphically

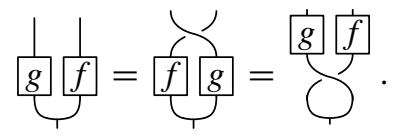

Dually, we say two morphisms $f: C \rightarrow V$ and $g: C \rightarrow W$ from a coalgebra $C$ in $\mathcal{B}$ comultiplication commute, or cocommute for short, and write $f \curlywedge g$ if

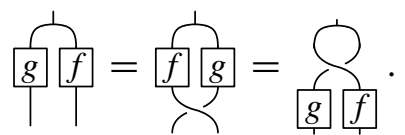

We say that $f, g$ bicommute if both $f \curlyvee g$ and $f \curlywedge g$.

If $A$ and $B$ are algebras in $\mathcal{B}$, then the natural maps $f: A \rightarrow A \otimes B$ and $g: B \rightarrow A \otimes B$ satisfy $f \curlyvee g$, but they only satisfy $g \curlyvee f$ if $A$ and $B$ are unbraided. In fact:

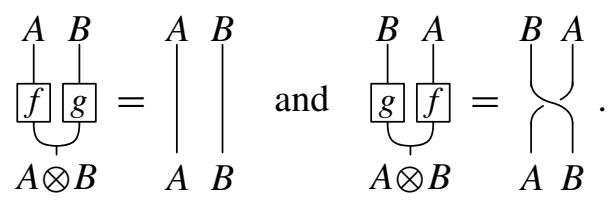

Lemma 2.1. Let $A$ be an algebra, $C$ a coalgebra, and $U, V, W, X, Y$ objects in $\mathcal{B}$.

(i) Let $f: U \rightarrow A, g: V \rightarrow A$ and $h: W \rightarrow A$.

(a) If $f \curlyvee g$ and $f \curlyvee h$, then $f \curlyvee(\nabla(g \otimes h))$.

(b) If $f \curlyvee h$ and $g \curlyvee h$, then $(\nabla(f \otimes g)) \curlyvee h$.

(c) If $f \curlyvee g$, then $f a \curlyvee g b$ for any $a: X \rightarrow U$ and $b: Y \rightarrow V$.

(ii) Let $f, g, h: C \rightarrow A$.

(a) If $f \curlyvee g$ and $f \curlyvee h$ then $f \curlyvee(g * h)$.

(b) If $f \curlyvee h$ and $g \curlyvee h$ then $(f * g) \curlyvee h$.

(c) If $f \curlyvee g$ and $g \curlywedge f$, then $f * g=g * f$.

(iii) Let $f, g: C \rightarrow A$.

(a) If $C$ is a bialgebra, $f, g$ are algebra morphisms, and $f \curlyvee g$, then $f * g$ is an algebra morphism.

(b) If $A, C$ are bialgebras, $f, g$ are bialgebra morphisms, $f \curlyvee g$ and $f \curlywedge g$, then $f * g$ is a bialgebra morphism.

(c) If $A$ is a bialgebra, $C$ a Hopf algebra, and $f$, $g$ are unital coalgebra morphisms, then $f \curlywedge g \Longleftrightarrow f * g$ is a coalgebra morphism. 
Note that $f \curlyvee g$ is not necessarily equivalent to $g \curlyvee f$ in the braided setting. The first part of the following result says, however, that the two properties are equivalent for Hopf algebras with sufficiently well-behaved antipodes. On the other hand, the second part says that if both properties are fulfilled then either the braiding is close to being a symmetry, or the morphisms are close to being trivial.

Proposition 2.2. Let $H, K$, and $A$ be Hopf algebras, and $f: H \rightarrow A, g: K \rightarrow A$ Hopf algebra morphisms.

(i) If $f \curlyvee g$, and if the antipode of $A$ is a monomorphism or the antipodes of $H$ and $K$ are epimorphisms, then $g \curlyvee f$.

(ii) If $f \curlyvee g$ and $g \curlyvee f$, then

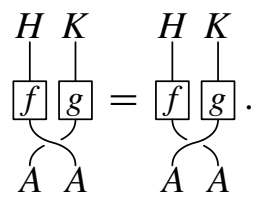

Proof. For the first claim, we calculate

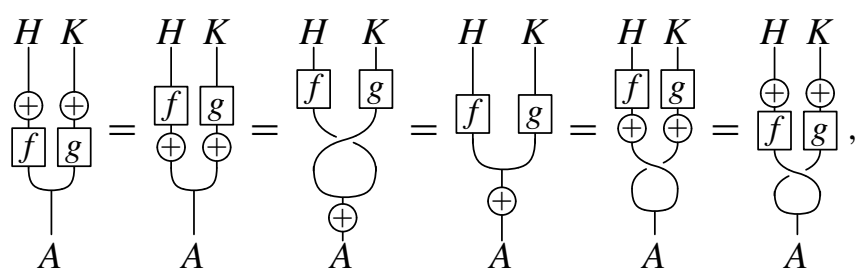

which implies $g \curlyvee f$ if the antipodes of $H$ and $K$ are epimorphisms. A similar argument shows the same if the antipode of $A$ is a monomorphism.

We now turn to the second claim. We have

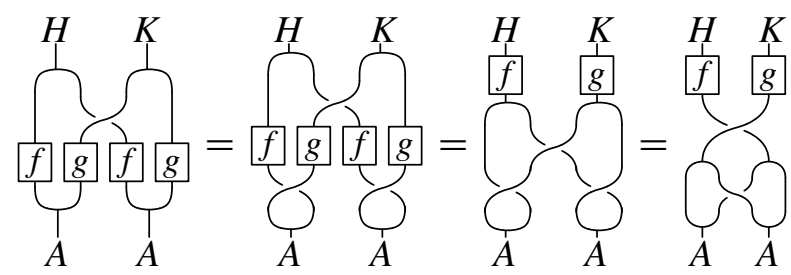

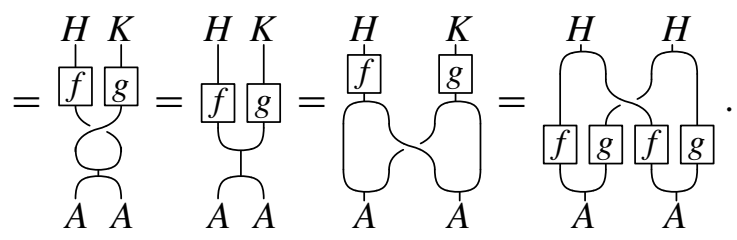


In other words,

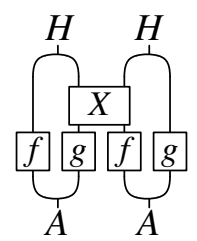

does not depend on the choice of $X \in\{>$,$\} . But since f \circ S$ and $g \circ S$ are convolution inverses to $f$ and $g$, respectively, we have

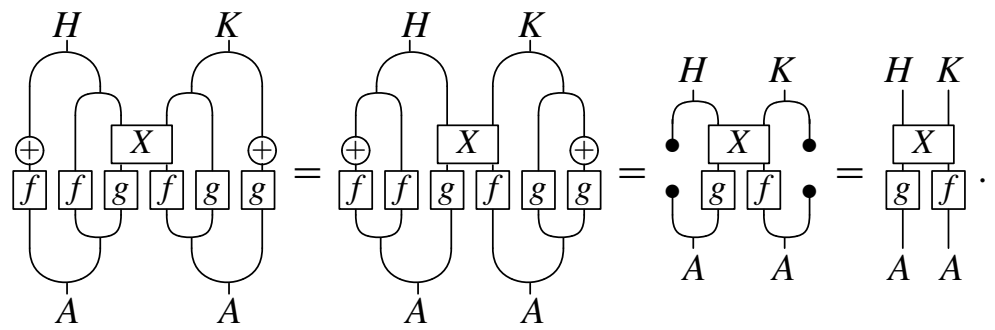

That the latter expression does not depend on the choice of $X$ is the claim.

As special cases one recovers two known facts that show how badly usual Hopf algebra constructions behave in a "truly braided" tensor category: a Hopf algebra cannot be commutative (or cocommutative) as a (co)algebra in $\mathcal{B}$ unless the braiding on the Hopf algebra is an involution [Schauenburg 1998], and the tensor product of two Hopf algebras cannot be a Hopf algebra unless the two factors are unbraided.

\section{Normal endomorphisms}

Recall that the left adjoint action and the left coadjoint coaction of a Hopf algebra $H$ on itself are
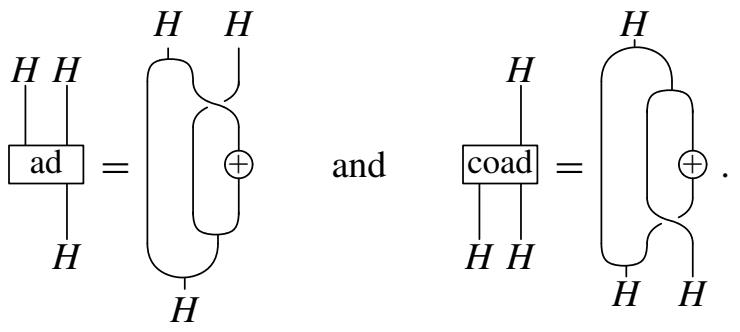

We note that the adjoint action is characterized by a twisted commutativity condition:

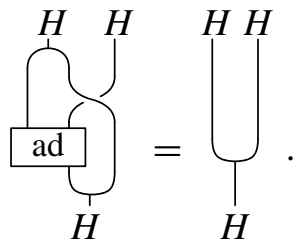


Definition 3.1. Let $f: H \rightarrow H$ be a morphism in $\mathcal{B}$, with $H$ a Hopf algebra.

(i) $f$ is normal if it is left $H$-linear with respect to the adjoint action.

(ii) $f$ is conormal if it is left $H$-colinear with respect to the coadjoint coaction.

(iii) $f$ is binormal if it is both normal and conormal.

For group algebras considered in the category of $\mathbb{C}$-vector spaces, the definition of a normal morphism agrees with the one used in group theory [Rotman 1995]. Since group algebras are cocommutative, every group endomorphism is trivially conormal. We will be primarily concerned with normal, conormal, and binormal endomorphisms of Hopf algebras.

Lemma 3.2. Let $f: H \rightarrow H$ be an endomorphism of the Hopf algebra $H$.

(i) The following are equivalent:

(a) $f$ is normal.

(b) $f \curlyvee\left((f S) * \operatorname{id}_{H}\right)$.

(c) $(f S) * \mathrm{id}_{H}$ is an algebra morphism.

(ii) The following are equivalent:

(a) $f$ is binormal.

(b) $f \curlywedge\left((f S) * \operatorname{id}_{H}\right)$ and $f \curlyvee\left((f S) * \mathrm{id}_{H}\right)$.

(c) $(f S) * \mathrm{id}_{H}$ is a bialgebra morphism

Proof. We only show part (i). For the equivalence of (b) and (c) we apply the bijection

$$
\mathcal{B}(H \otimes H, H) \ni T \mapsto \underbrace{H}_{H} \in \mathcal{B}(H \otimes H, H)
$$

to the two sides of the equation expressing multiplicativity of $g:=f S * \mathrm{id}$. We get

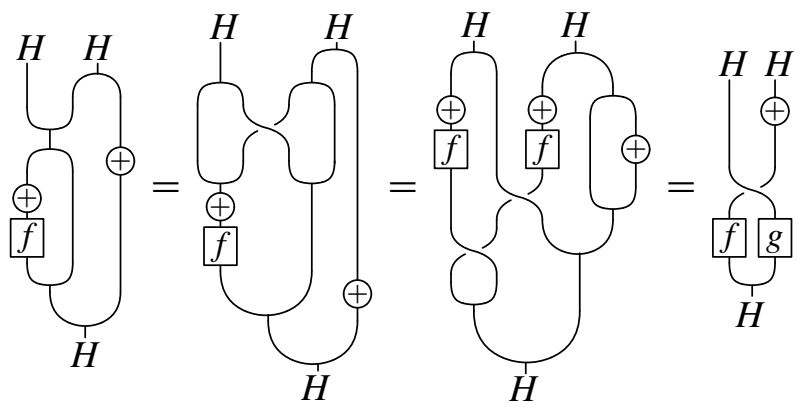




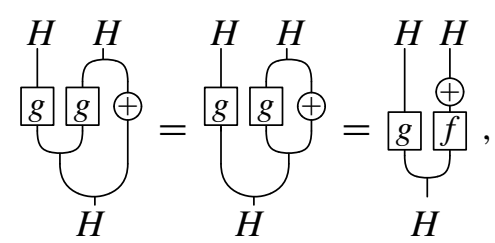

which are the two sides of (b), up to composition with the isomorphism $H \otimes S$.

For the equivalence of (a) and (b), we apply the bijection

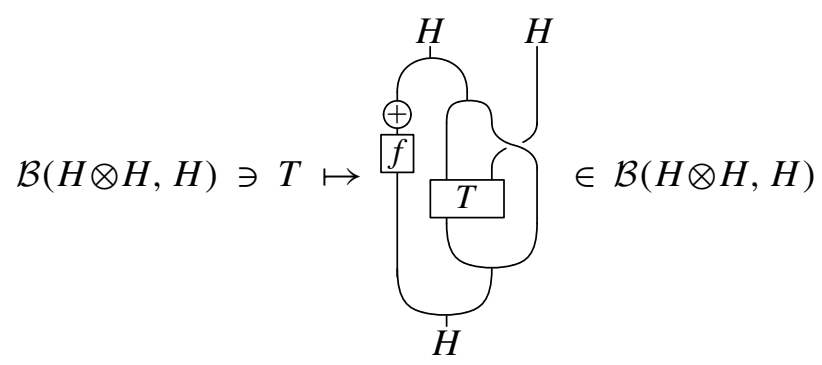

to the two sides of (a) and once again get two sides of (b):

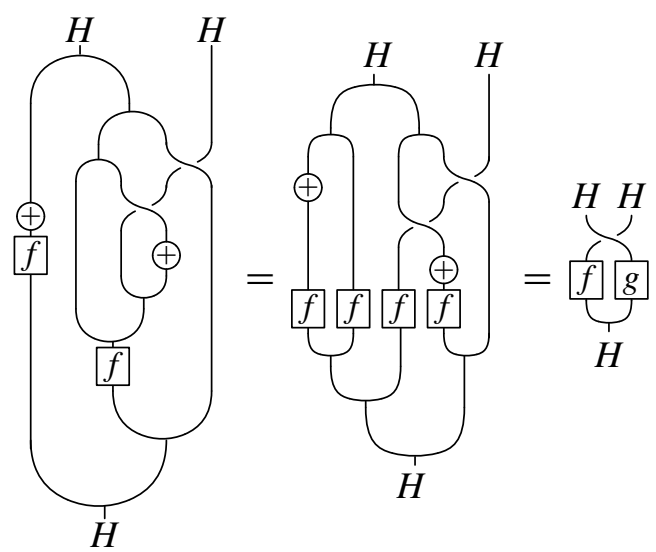

and

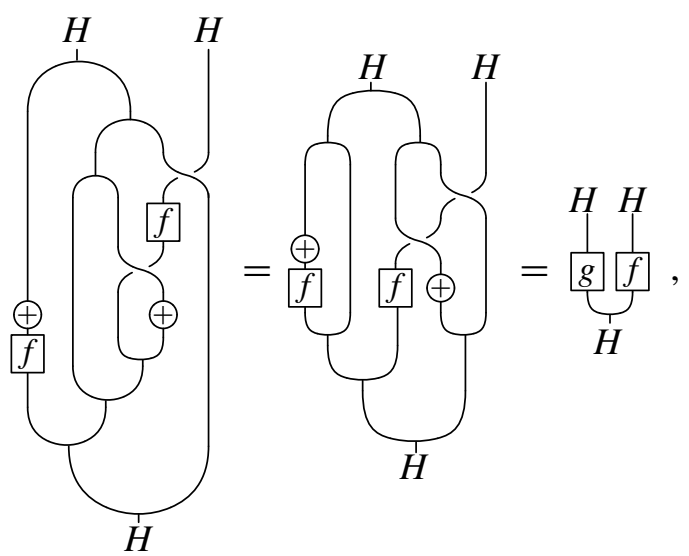




\section{Epic or monic endomorphisms}

We recall Radford's theorem [1985] on Hopf algebras with a projection, which was generalized to a categorical setting even more general than the one in the present paper by Bespalov and Drabant [1998]:

Theorem 4.1. Let $H$ be a Hopf algebra, and $\pi$ an idempotent Hopf algebra endomorphism of $H$. Then $H \cong \operatorname{Im}(\pi) \otimes \operatorname{Im}(p)$, where $p=(\pi \circ S) * \operatorname{id}_{H}$ is an idempotent endomorphism of the object $H$ in $\mathcal{B}$ (but not necessarily a Hopf endomorphism). $B:=\operatorname{Im}(p)$ is a subalgebra and a quotient coalgebra of $H$. The algebra structure of $\operatorname{Im}(\pi) \otimes B$ is a semidirect product with respect to a certain action of $K=\operatorname{Im}(\pi)$ on $B$, and the coalgebra structure is the cosemidirect product with respect to a certain coaction.

Moreover $\operatorname{Im}(p) \cong{ }^{\operatorname{co} \pi} H \cong \pi \backslash H$.

Proof. Only the last statement is not in [Bespalov and Drabant 1998], who avoid using coinvariant subobjects altogether to generalize [Radford 1985] to categories that might not have equalizers. We check the first isomorphism. We find

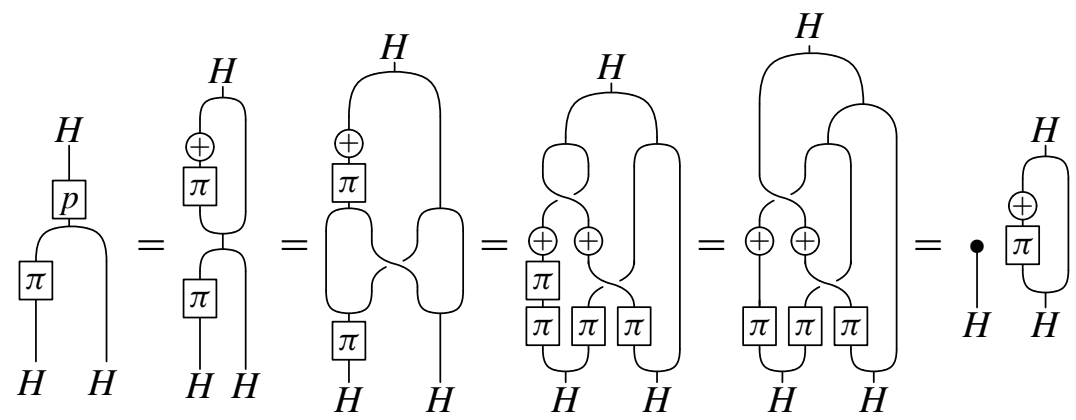

and if some morphism $t: T \rightarrow H$ satisfies $\left(\pi \otimes \operatorname{id}_{H}\right) \Delta t=\eta \otimes t$, then

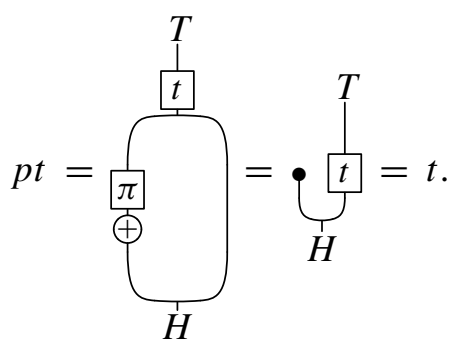

Proposition 4.2. Let $H$ be a Hopf algebra, and $f$ a Hopf algebra endomorphism of $H$. Assume that $H$ satisfies both chain conditions. Then there is $n \in \mathbb{N}$ such that $H \cong \operatorname{Im}\left(f^{n}\right) \otimes{ }^{\operatorname{co} f^{n}} H$ is a Radford biproduct.

Proof. Consider the epi-mono factorization $f=(H \stackrel{e}{\rightarrow} B \stackrel{m}{\rightarrow} H)$, where we identify $B=\operatorname{Im}(f)=\operatorname{Coim}(f)$. Then the endomorphism $t=e m$ of $B$ satisfies $m t=f m$ and $t e=e f$. The chain conditions on $H$ imply that the ascending chain of the 
kernels of $f^{n}$ and the descending chain of the images, hence the ordered chain of quotient objects formed by the cokernels of $f^{n}$ stabilize. Then, replacing $f$ by a suitable power $f^{n}$, we can assume that $t$ is an isomorphism. Then $\pi=m t^{-1} e$ is an idempotent endomorphism of $H$, since $\pi^{2}=m t^{-1} e m t^{-1} e=m t^{-1} t t^{-1} e=$ $m t^{-1} e=\pi$.

Thus $H \cong \operatorname{Im}(\pi) \otimes{ }^{c o \pi} H$ is a Radford biproduct. Moreover, $\operatorname{Im}(\pi)=\operatorname{Im}(f)$, and ${ }^{\operatorname{co} \pi} H={ }^{\operatorname{co} f} H$.

Proposition 4.3. Let $H$ be a Hopf algebra in $\mathcal{B}$ that satisfies both chain conditions, and $f$ a Hopf algebra endomorphism of $H$.

(i) If the left or right $f$-coinvariants of $H$ are trivial, then $f$ is a monomorphism in $\mathcal{B}$.

(ii) If the left or right $f$-invariant quotient of $H$ is trivial, then $f$ is an epimorphism in $\mathcal{B}$.

Proof. We prove the first part. By Proposition 4.2, $H \cong \operatorname{Im}\left(f^{n}\right) \otimes{ }^{\operatorname{co}} f^{n} H$ is a Radford

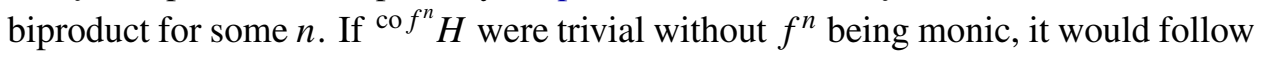
that $H$ is isomorphic to a proper quotient of itself, contradicting the chain conditions. Now assume for some $m>1$ that ${ }^{\text {co } f^{m}} H$ is nontrivial. Let $j:$ co $f^{m} H \rightarrow H$ be the inclusion. By exactness of tensor products in $\mathcal{B}$, we have an equalizer

$$
0 \longrightarrow{ }^{\operatorname{co} f} H \otimes H \longrightarrow H \underset{\eta \otimes H \otimes H}{\stackrel{(f \otimes H) \Delta \otimes H}{\longrightarrow}} H \otimes H \otimes H
$$

and by the calculation

$$
\begin{aligned}
\left(\left(f^{m-1} \otimes H\right) \Delta \otimes H\right)(f \otimes H) \Delta j & =\left(f^{m} \otimes f \otimes H\right)(\Delta \otimes H) \Delta j \\
& =\left(f^{m} \otimes(f \otimes H) \Delta\right) \Delta j=\eta \otimes(f \otimes H) \Delta j
\end{aligned}
$$

we see that $(f \otimes H) \Delta j$ factors through this equalizer. We conclude that if $(f \otimes H) \Delta j$ were not trivial, then it would follow that ${ }^{\operatorname{co} f^{m-1}} H \otimes H$ is not $I \otimes H$, which implies that ${ }^{\operatorname{co} f^{m-1}} H$ is nontrivial. We can conclude by induction that ${ }^{\operatorname{co} f} H$ is nontrivial after all.

Remarks 4.4. Let $f: H \rightarrow G$ be a Hopf algebra homomorphism in $\mathcal{B}$.

(i) Clearly, if $f$ is a monomorphism in $\mathcal{B}$, then it is a monomorphism in $\underline{\operatorname{Hopf} A \lg }(\mathcal{B})$.

(ii) If $f$ has trivial left or right coinvariants, then $f$ is a monomorphism in Coalg $(\mathcal{B})$.

(iii) If $f$ is normal, and a monomorphism in $\operatorname{Hopf} \operatorname{Alg}(\mathcal{B})$, then $f$ has trivial left and right coinvariants.

Thus the preceding result shows that normal endomorphisms of a Hopf algebra in $\mathcal{B}$ satisfying both chain conditions are monic (epic) if and only if they are so considered as morphisms in $\mathcal{B}$. 
Proof. If $C$ is a coalgebra and $g, h: C \rightarrow H$ are coalgebra morphisms with $f g=f h$, then

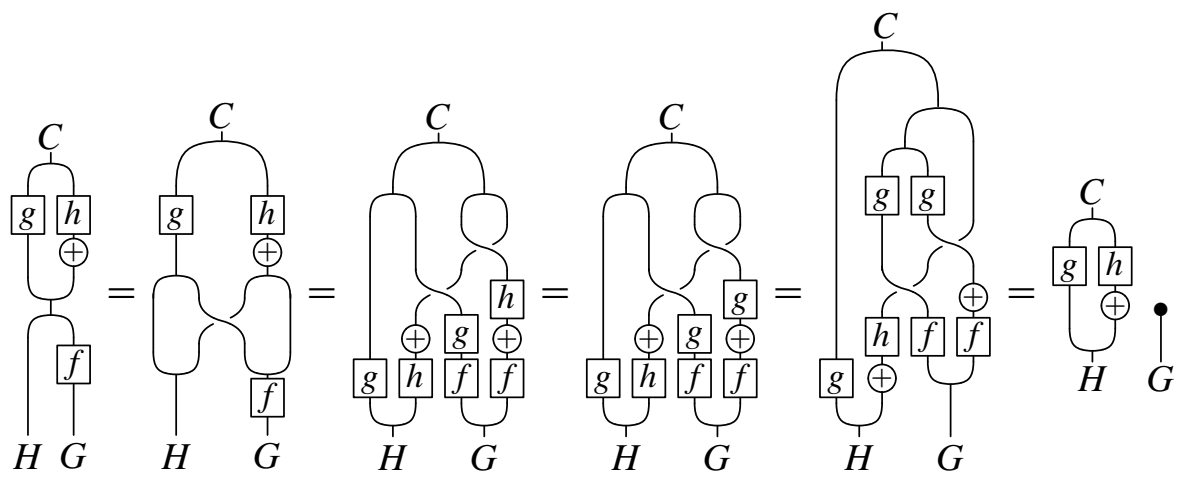

and thus, if $H^{\operatorname{co} f}$ is trivial, $g * S h=\eta \varepsilon$, whence $g=h$. If $f$ is normal, then the coinvariants are a Hopf subalgebra.

Remark 4.5. In general it is false that monic is equivalent to trivial coinvariants, or that epic is equivalent to trivial invariants. In finite dimensions these concepts agree by the Nichols-Zoeller theorem [1989]; see also [Scharfschwerdt 2001]. In infinite dimensions, however, counterexamples are known [Chirvăsitu 2010].

Lemma 4.6. Let $H$ be a Hopf algebra in $\mathcal{B}$ that satisfies both chain conditions. Assume further that the braiding $\tau_{H H}$ has finite order. Then the antipode of $H$ is an automorphism in $\mathcal{B}$.

Proof. Depict the iterates of the antipode by

$$
S^{m}=\stackrel{1}{T} \text {. }
$$

One has

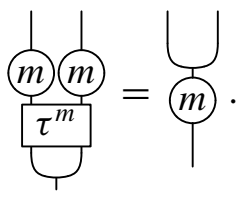

Using this, we can show inductively that the coinvariants of $H$ under an iterate of the antipode are trivial as follows. Let $t: T \rightarrow H$ be a morphism factoring through ${ }^{c o} S^{2 n} H$, i.e., $\left(S^{2 n} \otimes H\right) \Delta t=\eta \otimes t$. We will show that $\left(S^{m} \otimes H\right) \Delta t=\eta \otimes t$ for any $m$, whence (taking $m=0) t=\eta \varepsilon t$. 
Assume $\left(S^{m+1} \otimes H\right) \Delta t=\eta \otimes t$, or pictorially

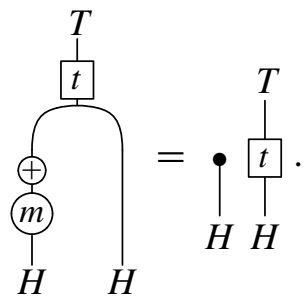

Then

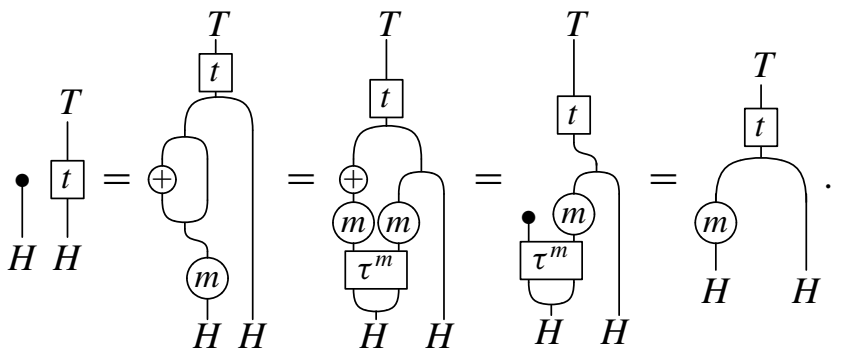

Since the braiding on $H$ has finite order by assumption, some even power of the antipode is a Hopf algebra endomorphism of $H$. Therefore that even power of the antipode is a monomorphism in $\mathcal{B}$. By the dual reasoning it is also an epimorphism, and therefore $S$ itself is an automorphism in $\mathcal{B}$.

\section{Fitting's lemma}

Proposition 5.1 (Fitting's lemma). Let $H$ be a Hopf algebra, and $f$ a Hopf algebra endomorphism of $H$. Assume that $H$ satisfies both chain conditions, so that there is an $n \in \mathbb{N}$ such that $H \cong \operatorname{Im}\left(f^{n}\right) \otimes{ }^{\operatorname{co} f^{n}} H$ is a Radford biproduct.

If $f$ is normal, the action of $\operatorname{Im}\left(f^{n}\right)$ on ${ }^{\operatorname{co} f^{n}} H$ is trivial, so that, as an algebra, $H$ is the tensor product of $\operatorname{Im}\left(f^{n}\right)$ and ${ }^{\operatorname{co}^{n}} H$ in $\mathcal{B}$. Similarly if $f$ is conormal, then the coalgebra $H$ is a tensor product of coalgebras in $\mathcal{B}$. In particular, if $f$ is binormal then $\operatorname{Im}\left(f^{n}\right)$ and ${ }^{\operatorname{co} f^{n}} H$ are unbraided Hopf algebras in $\mathcal{B}$, and $H$ is isomorphic to their tensor product.

Proof. We continue the proof of Proposition 4.2, assuming that $\operatorname{Ker}\left(f^{2}\right)=\operatorname{Ker}(f)$ and $\operatorname{Coker}\left(f^{2}\right)=\operatorname{Coker}(f)$ after replacing $f$ by a power of $f$. We now add the observation that normality of $f$ implies that $p=\left(f *\left(S f * \mathrm{id}_{H}\right)\right) p=\left(S f * \mathrm{id}_{H}\right) p$. Therefore $f \curlyvee p$, and dually $f \curlywedge p$ if $f$ is conormal. This in turn implies that the Radford biproduct is just an ordinary tensor product algebra or tensor product coalgebra, as appropriate.

Definition 5.2. Let $H$ be a Hopf algebra. If $H \cong A \otimes B$ for two Hopf algebras $A$ and $B$, we say that $A$ is a tensor factor of $H$. (This implies that $A$ and $B$ are unbraided.) We say that $H$ is tensor indecomposable if it does not have a nontrivial tensor factor. An endomorphism $f$ of $H$ is nilpotent if there is an $n \in \mathbb{N}$ such that $f^{n}=\eta \varepsilon$. 
Corollary 5.3. If $H$ is a tensor indecomposable Hopf algebra satisfying both chain conditions, then every binormal endomorphism of $H$ is nilpotent or an automorphism.

\section{Krull-Remak-Schmidt}

Of course, a Hopf algebra satisfying both chain conditions can be (inductively) decomposed as a tensor product of indecomposable Hopf subalgebras. We shall now show that the Hopf algebraic analog of the Krull-Remak-Schmidt theorem asserting the uniqueness of such a decomposition also holds. A version of this for completely reducible semisimple Hopf algebras was established in [Burciu 2011]. In general, it cannot be hoped that this result has a categorical version. In [Müger 2003] it was shown that a nondegenerate fusion category factorizes into a product of prime ones, but that this was generally not unique. Therefore, such decompositions are rather specific to Hopf algebras.

Lemma 6.1. Let $f, g$ be bicommuting, binormal endomorphisms of a tensor indecomposable Hopf algebra $\mathrm{H}$.

If $f$ and $g$ are nilpotent, then so is $f * g$.

Proof. Otherwise $f * g$ is a normal automorphism, and after composing $f$ and $g$ with its inverse, we can assume that $f * g=\mathrm{id}_{H}$. In particular $f$ composition commutes with $g$. Then one can show by induction that $\operatorname{id}_{H}=(f * g)^{n}$ is a convolution product of terms of the form $f^{k} g^{n-k}$ for $0 \leq k \leq n$ (in fact this is a binomial formula with binomial coefficients, but writing it is cumbersome because addition is replaced with convolution products). If $f^{m}=\eta \varepsilon=g^{m}$, this implies $(f * g)^{2 m}=\eta \varepsilon$, since each term contains an $m$-th power of either $f$ or $g$.

Remark 6.2. Let $H$ and $H_{1}, \ldots, H_{k}$ be Hopf algebras in $\mathcal{B}$. Decomposing $H$ as a tensor product Hopf algebra

$$
H \cong H_{1} \otimes H_{2} \otimes \cdots \otimes H_{k}
$$

amounts to specifying a system of injections $\iota_{i}: H_{i} \rightarrow H$ and projections $\pi_{i}: H \rightarrow H_{i}$, all of them Hopf algebra morphisms, which commute and cocommute pairwise and satisfy $\pi_{i} \iota_{i}=\mathrm{id}_{H_{i}}, \pi_{i} \iota_{j}=\eta \varepsilon$ if $i \neq j$, and $\iota_{1} \pi_{1} * \iota_{2} \pi_{2} * \cdots * \iota_{k} \pi_{k}=\mathrm{id}_{H}$. The isomorphisms between $H$ and the tensor product are then given by

$$
H \stackrel{\Delta^{(k-1)}}{\longrightarrow} H^{\otimes k} \stackrel{\pi_{1} \otimes \cdots \pi_{k}}{\longrightarrow} H_{1} \otimes \cdots \otimes H_{k}
$$

and

$$
H_{1} \otimes \cdots \otimes H_{k} \stackrel{\iota_{1} \otimes \cdots \otimes \iota_{k}}{\longrightarrow} H^{\otimes k} \stackrel{\nabla^{(k-1)}}{\longrightarrow} H .
$$

Note that the $H_{i}$ need to be pairwise unbraided for the tensor product Hopf algebra to make sense. 
Theorem 6.3. Let $H$ be a Hopf algebra in $\mathcal{B}$, and let

$$
\begin{aligned}
H & =H_{1} \otimes H_{2} \otimes \cdots \otimes H_{k} \\
& =G_{1} \otimes G_{2} \otimes \cdots \otimes G_{\ell}
\end{aligned}
$$

be two tensor decompositions of $H$ in tensor indecomposable factors.

Then $k=\ell$, and $H_{i} \cong G_{i}$ after a suitable permutation of the indices.

Moreover, letting

$$
H_{i} \underset{\pi_{i}}{\stackrel{\iota_{i}}{\rightleftarrows}} H \underset{q_{j}}{\stackrel{p_{j}}{\rightleftarrows}} G_{j}
$$

denote the systems of injections and projections associated to the decompositions into tensor factors, then the factors can be so numbered that for any $1 \leq m \leq k$

$$
H \stackrel{\Delta^{(k-1)}}{\longrightarrow} H^{\otimes k} \stackrel{\pi_{1} \otimes \cdots \otimes \pi_{m} \otimes p_{m+1} \otimes \cdots \otimes p_{k}}{\longrightarrow} H_{1} \otimes \cdots \otimes H_{m} \otimes G_{m+1} \otimes \cdots \otimes G_{k}
$$

and

$$
H_{1} \otimes \cdots \otimes H_{m} \otimes G_{m+1} \otimes \cdots \otimes G_{k} \stackrel{\iota_{1} \otimes \cdots \otimes \iota_{m} \otimes q_{m+1} \otimes \cdots \otimes q_{k}}{\longrightarrow} H^{\otimes k} \stackrel{\nabla^{(k-1)}}{\longrightarrow} H
$$

are isomorphisms.

Proof. There is nothing to show if one of the decompositions consists of only one factor. Otherwise we consider

$$
\operatorname{id}_{H_{1}}=\pi_{1} \iota_{1}=\pi_{1}\left(q_{1} p_{1} * \cdots * q_{\ell} p_{\ell}\right) \iota_{1}=\pi_{1} q_{1} p_{1} \iota_{1} * \cdots * \pi_{1} q_{\ell} p_{\ell} \iota_{1} .
$$

Since $H_{1}$ is indecomposable, and the terms in the last convolution product are bicommuting binormal endomorphisms, we know that one of $\pi_{1} q_{j} p_{j} \iota_{1}$ is an isomorphism. Without loss of generality we assume this happens for $j=1$, and that $\pi_{1} q_{1} p_{1} \iota_{1}=\mathrm{id}_{H_{1}}$. It follows that $\pi_{1} q_{1}$ and $p_{1} \iota_{1}$ are mutually inverse isomorphisms between $H_{1}$ and $G_{1}$. Now put $f=q_{2} p_{2} * \cdots * q_{\ell} p_{\ell}$ and $t=\iota_{1} \pi_{1} q_{1} p_{1} * f$. Since $p_{1} t=p_{1} \iota_{1} \pi_{1} q_{1} p_{1}=p_{1}$, we have $H^{\text {cot }} \subset H^{\text {co } p_{1} t}=H^{\text {co } p_{1}}$. Thus, for $j: H^{\text {cot} t} \rightarrow H$ the inclusion, we find

$$
\Delta j=\left(H \otimes q_{1} p_{1} * \cdots * q_{\ell} p_{\ell}\right) \Delta j=(H \otimes f) \Delta j=(H \otimes t) \Delta j=(H \otimes \eta) j,
$$

and therefore $H^{\operatorname{co} t}$ is trivial. We conclude that $t$ is an automorphism of $H$.

Write $\tilde{\pi}: H \rightarrow H_{2} \otimes \cdots \otimes H_{k}=: \tilde{H}$ and $\tilde{\imath}: \tilde{H} \rightarrow H$ for the natural projection and injection morphisms, and similarly for $\tilde{p}: H \rightarrow \tilde{G}, \tilde{q}: \tilde{G} \rightarrow H$. Since $t q_{1}=\iota_{1} \pi_{1} q_{1}$, we have $\tilde{\pi} t q_{1}=\eta \varepsilon$, and thus $\tilde{\pi} t=\tilde{\pi} t \tilde{q} \tilde{p}$ and $\tilde{\pi} t \tilde{q} \tilde{p} t^{-1} \tilde{\iota}=\tilde{\pi} \tilde{\iota}=\mathrm{id}_{\tilde{H}}$. It follows that $\tilde{\pi} t \tilde{q}$ and $\tilde{p} t^{-1} \tilde{\iota}$ are mutually inverse isomorphism between $\tilde{G}$ and $\tilde{H}$.

Thus, by an inductive argument we have $k=\ell$, and we can rearrange the indices to get $H_{i} \cong G_{i}$ for all $i$. 
Note further that the automorphism $t$ above is the composition of the isomorphism

$$
H \stackrel{\Delta^{(k-1)}}{\longrightarrow} H^{\otimes k} \stackrel{p_{1} \otimes \cdots \otimes p_{k}}{\longrightarrow} G_{1} \otimes \cdots \otimes G_{k} \stackrel{\pi_{1} q_{1} \otimes G_{2} \otimes \cdots \otimes G_{k}}{\longrightarrow} H_{1} \otimes G_{2} \otimes \cdots \otimes G_{k}
$$

with the morphism

$$
H_{1} \otimes G_{2} \otimes \cdots \otimes G_{k} \stackrel{\iota_{1} \otimes q_{2} \otimes \cdots \otimes q_{k}}{\longrightarrow} H^{\otimes k} \stackrel{\nabla^{(k-1)}}{\longrightarrow} H,
$$

whence the latter is an isomorphism. Again by an inductive argument, we get that (6-2) is an isomorphism; the reasoning for (6-1) is similar.

\section{Endomorphisms of tensor products}

Let $H$ and $K$ be two Hopf algebras in $\mathcal{B}$, unbraided so that one can form the tensor product bialgebra $H \otimes K$. Let $A$ be an algebra in $\mathcal{B}$. It is well known that there is a bijection

$$
\underline{\operatorname{Alg}}(H \otimes K, A) \cong\{(a, b) \in \underline{\operatorname{Alg}}(H, A) \times \underline{\operatorname{Alg}}(K, A) \mid a \curlyvee b\} .
$$

In fact, a pair $(a, b)$ of multiplication commuting algebra morphisms induces $f=\nabla_{A}(a \otimes b)$, and

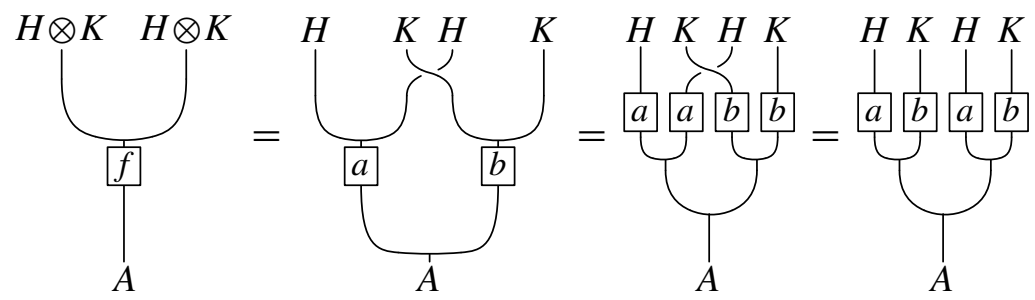

shows that $f$ is multiplicative. Conversely, given $f: H \otimes K \rightarrow A$, define $a=$ $f(H \otimes \eta)$ and $b=f(\eta \otimes K)$. Then, with $T:=H \otimes K$ :

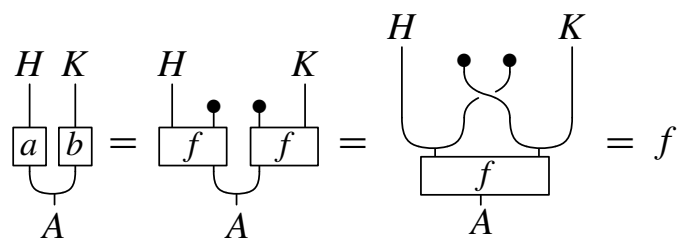

and

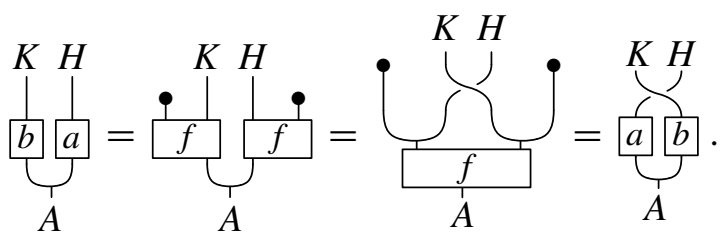

Assume that $A$ is a bialgebra in $\mathcal{B}$, and $a, b, f$ are as above. Then $f$ is a bialgebra homomorphism if and only if $a$ and $b$ are. 
Dually, for a coalgebra $C$ in $\mathcal{B}$, a bijection

$$
\{(a, b) \in \underline{\operatorname{Coalg}}(C, H) \times \underline{\operatorname{Coalg}}(C, H) \mid a \curlywedge b\} \longrightarrow \underline{\operatorname{Coalg}}(C, H \otimes K)
$$

is given by $(a, b) \mapsto(a \otimes b) \Delta$, and it induces bijections on the subsets containing (pairs of) bialgebra maps.

Putting the above together, one obtains a bijection between $\operatorname{End}(H \otimes K)$ and

$$
\left\{\begin{array}{l|l}
(a, b, c, d) & \begin{array}{l}
a \in \operatorname{End}(H), b \in \operatorname{Hom}(K, H), \\
c \in \operatorname{Hom}(H, K), d \in \operatorname{End}(K), \\
a \curlyvee b, c \curlyvee d, a \curlywedge c, b \curlywedge d
\end{array}
\end{array}\right\}
$$

with the endomorphism of $H \otimes K$ corresponding to a quadruple of Hopf algebra map "components" given by

$$
\left(\begin{array}{ll}
a & b \\
c & d
\end{array}\right):=\underbrace{C_{a}}_{H}
$$

Consider a second endomorphism $g$ of $H \otimes K$ dissected analogously into a matrix $\left(\begin{array}{ll}a^{\prime} & b^{\prime} \\ c^{\prime} & d^{\prime}\end{array}\right)$ of Hopf algebra endomorphisms. Then it is straightforward to check that $g f$ corresponds to $\left(\begin{array}{ll}a^{\prime} a * b^{\prime} c & a^{\prime} b * b^{\prime} d \\ c^{\prime} a * d^{\prime} c & c^{\prime} b * d^{\prime} d\end{array}\right)$.

Proposition 7.1. Let $H$ and $K$ be as above, and $f \in \operatorname{End}(H \otimes K)$ described by a matrix $\left(\begin{array}{ll}a & b \\ c & d\end{array}\right)$. Assume that the antipodes of $H$ and $K$ are automorphisms in $\mathcal{B}$.

Then $f$ is normal if and only if $a$ and $c$ are normal, $b \curlyvee \mathrm{id}_{H}$, and $d \curlyvee \mathrm{id}_{K} ; a$ similar characterization holds for conormal endomorphisms.

Proof. We fix projections and injections for the tensor product $P:=H \otimes K$ :

$$
H \underset{\pi_{H}}{\stackrel{\iota_{H}}{\rightleftarrows}} P \underset{\iota_{K}}{\stackrel{\pi_{K}}{\rightleftarrows}} K
$$

First assume that $f$ is normal. Since $f \curlyvee\left(f S * \operatorname{id}_{P}\right), a=\pi_{H} f \iota_{H}$ commutes with $\pi_{H}\left(f S * \operatorname{id}_{P}\right) \iota_{H}=a S * \operatorname{id}_{H}$. Similarly $c$ is normal. Using (3-1) we have

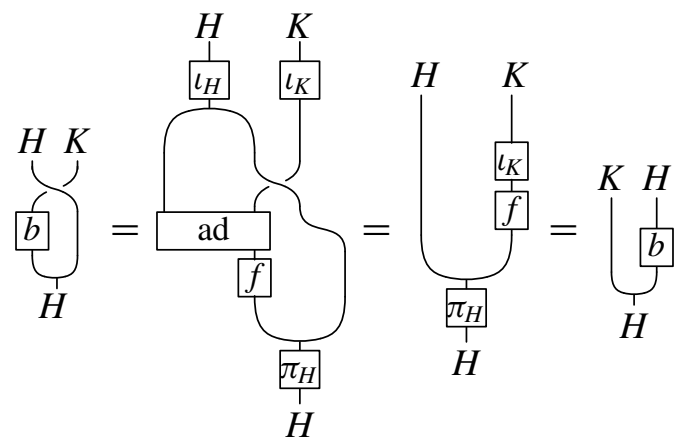

so that $b \curlyvee \mathrm{id}_{H}$. Similarly $d \curlyvee \mathrm{id}_{K}$. 
Now suppose that the stated normality and commutation conditions on $a, b, c, d$ hold. Writing $\hat{a}=\iota_{H} a \pi_{H}, \hat{b}=\iota_{H} b \pi_{K}$ etc. we can write $f=\hat{a} * \hat{b} * \hat{c} * \hat{d}$ as a convolution product of four commuting and cocommuting endomorphisms of $P$. We are claiming that this product commutes with

$$
f S * \operatorname{id}_{P}=\hat{a} S * \hat{b} S * \hat{c} S * \hat{d} S * \iota_{K} \pi_{K} * \iota_{H} \pi_{H}=\hat{b} S * \hat{c} S * \hat{d} S * \iota_{K} \pi_{K} * \hat{a} S * \iota_{H} \pi_{H} .
$$

(the last equality using that $\hat{a}$ bicommutes with $\hat{b}, \hat{c}, \hat{d}$, and $\iota_{K}$.) Now $\hat{a}$ commutes with $\hat{a} S * \iota_{H} \pi_{H}$ since $a$ is normal, with $\hat{b} S$ since $a \curlyvee b$, and with $\iota_{K} \pi_{K}$ and $\hat{c} S$ since $\iota_{H} \curlyvee \iota_{K}$. The next factor $\hat{b}$ commutes with $\hat{a} S, \hat{b} S$, and $\iota_{H} \pi_{H}$ since $b \curlyvee \operatorname{id}_{H}$, and it commutes with $\hat{c} S, \hat{d} S$, and $\iota_{K} \pi_{K}$, since $\iota_{K} \curlyvee \iota_{H}$. Similar arguments deal with the convolution factors $\hat{c}$ and $\hat{d}$.

Remark 7.2. Similarly, an endomorphism $f$ of a tensor product of several pairwise unbraided Hopf algebras $H_{1}, \ldots, H_{k}$ can be described by a matrix $\left(v_{i j}\right)$ of Hopf algebra homomorphisms between the factors. By inductive arguments one can show that $f$ is normal if and only if all the diagonal terms are normal, and the off-diagonal terms commute with the identities on their targets.

An interesting case arises when there are no nontrivial homomorphisms $H_{i} \rightarrow H_{j}$ commuting with $\mathrm{id}_{H_{j}}$. In this case any normal endomorphism preserves the decomposition into tensor factors. One can deduce from this that the Krull-RemakSchmidt decomposition is unique in a stronger sense than up to permutation and isomorphism; in the original case of decompositions of groups the uniqueness of the subgroups in a direct decomposition into directly indecomposable factors follows as stated in Remak's thesis [1911].

\section{Automorphisms of tensor products}

We consider now the automorphisms of tensor products of Hopf algebras. These are the natural extensions of the corresponding results in group theory [Bidwell et al. 2006; Bidwell 2008].

Throughout this section we let $H$ and $K$ be unbraided Hopf algebras, so that we can form the tensor product $H \otimes K$, and we assume that the antipodes of $H$ and $K$ are automorphisms in $\mathcal{B}$.

Identify endomorphisms of $H \otimes K$ with matrices of Hopf algebra homomorphisms as in Section 7. Let $\left(\begin{array}{ll}a & b \\ c & d\end{array}\right) \in \operatorname{End}(H \otimes K)$. If $a$ is an automorphism, then by (c) of Lemma 2.1 the condition $a \curlyvee b$ implies id $\operatorname{id}_{H} \curlyvee b$ (and $x \curlyvee b$ for any $x: X \rightarrow H$ ). Similarly $\mathrm{id}_{K} \curlywedge c$, and, if $d$ is also an automorphism, then $b \curlywedge \mathrm{id}_{H}$ and $c \curlyvee \mathrm{id}_{K}$.

Define

$$
\mathcal{A}=\left(\begin{array}{cc}
\operatorname{Aut}(H) & \operatorname{Hom}^{c}(K, H) \\
\operatorname{Hom}^{c}(H, K) & \operatorname{Aut}(K)
\end{array}\right),
$$


where $\operatorname{Hom}^{c}(K, H):=\left\{b \in \operatorname{Hom}(K, H) \mid b \curlyvee \mathrm{id}_{H}\right.$ and $\left.b \curlywedge \mathrm{id}_{H}\right\}$. This is easily seen to be an abelian group under convolution product. Indeed, the image of any such morphism determines an abelian Hopf subalgebra of $H$. Note that $b \curlyvee \operatorname{id}_{H}$ $\Longleftrightarrow b \curlyvee \alpha$ for some/all $\alpha \in \operatorname{Aut}(H)$, and similarly $b \curlywedge$ id $\Longleftrightarrow b \curlywedge \alpha$ for some/all $\alpha \in \operatorname{Aut}(H)$.

Consider an automorphism $f$ of $H \otimes K$, and its decomposition as a matrix $\left(\begin{array}{ll}a & b \\ c & d\end{array}\right)$ of Hopf algebra homomorphisms as in Section 7. Let $f^{-1}$ correspond in the same way to a matrix $\left(\begin{array}{cc}a^{\prime} & b^{\prime} \\ c^{\prime} & d^{\prime}\end{array}\right)$. Then we have $\operatorname{id}_{K}=(\varepsilon \otimes K) f^{-1} f(\eta \otimes K)=c^{\prime} b * d^{\prime} d$. Since $c^{\prime} \curlyvee d^{\prime}$ and $b \curlywedge d$, we have that $c^{\prime} b \curlyvee d^{\prime} d=\left(c^{\prime} b S\right) * \operatorname{id}_{K}$ and $c^{\prime} b \curlywedge\left(c^{\prime} b S\right) * \operatorname{id}_{K}$. In other words, $c^{\prime} b$ is a binormal endomorphism of $K$. In the same way $b c^{\prime}$ is a binormal endomorphism of $H$. If we assume both chain conditions on $H$ and $K$, then for $n$ sufficiently large $b$ and $c^{\prime}$ induce mutually inverse isomorphisms between the images of $\left(c^{\prime} b\right)^{n}$ and $\left(b c^{\prime}\right)^{n}$. Thus, using Fitting's lemma, the image of $\left(c^{\prime} b\right)^{n}$ is a common tensor factor of $H$ and $K$.

This gives part of the following result.

Theorem 8.1. Suppose that $H$ and $K$ satisfy both chain conditions. Then $\mathcal{A} \subseteq$ $\operatorname{Aut}(H \otimes K)$ if and only if $H$ and $K$ have no nontrivial common abelian direct tensor factors. On the other hand, $\operatorname{Aut}(H \otimes K)=\mathcal{A}$ if and only if $H$ and $K$ have no nontrivial common direct tensor factors.

Proof. If $H$ and $K$ have a common nontrivial direct tensor factor, then permutations of this factor in $H \otimes K$ are automorphisms of $H \otimes K$ not contained in $\mathcal{A}$.

By the preceding remarks, to show $\operatorname{Aut}(H \otimes K) \subseteq \mathcal{A}$ it remains to prove that the common tensor factor in $H \otimes K$ that we found is necessarily nontrivial if $d$ is not an automorphism. A similar argument will apply to show that $a$ is an automorphism, and the commutation and cocommutation conditions for the components of an endomorphism will be equivalent to the off-diagonal terms (co)commuting with the identity instead of the automorphisms on the diagonal.

Thus suppose that $d$ is not an automorphism. Then we can assume without loss of generality that the right $d$-coinvariant subobject $D$ of $H$ is nontrivial. If $\iota: D \rightarrow H$ is the inclusion, then $c^{\prime} b \iota=\nabla\left(c^{\prime} b \otimes \eta\right) \iota=\nabla\left(c^{\prime} b \otimes d^{\prime} d\right) \Delta \iota=\left(c^{\prime} b * d^{\prime} d\right) \iota=\iota$, hence $\left(c^{\prime} b\right)^{n} \iota=\iota$ for all $n$, and the image of $\left(c^{\prime} b\right)^{n}$ is nontrivial as desired.

The desired equality in the second part will then hold once we have proven the first equivalence.

To this end we first consider the forward direction by contrapositive. Suppose that $H$ and $K$ have a common abelian direct tensor factor $L$, and write $H=H^{\prime} \otimes L$ and $K=K^{\prime} \otimes L$. Since $L$ is abelian, its antipode $S_{L}$ is a Hopf endomorphism of $L$. Taking $a=\mathrm{id}_{H}, d=\mathrm{id}_{K}, b=\eta_{K^{\prime}} \varepsilon_{K^{\prime}} \otimes S_{L}$ and $c=\eta_{H^{\prime}} \varepsilon_{H^{\prime}} \otimes S_{L}$ we find that $\psi=\left(\begin{array}{ll}a & b \\ c & d\end{array}\right) \in \mathcal{A}$. However, $L$ is a subobject of the right $\psi$-coinvariant subobject, whence $\psi \notin \operatorname{Aut}(H \otimes K)$. 
For the remaining direction, assume that $f=\left(\begin{array}{ll}a & b \\ c & d\end{array}\right)$ belongs to $\mathcal{A}$; in particular $f$ is a Hopf algebra endomorphism of $H \otimes K$. After multiplying with the obvious automorphism of $H \otimes K$, namely $\left(\begin{array}{cc}a^{-1} & \eta \varepsilon \\ \eta \varepsilon & d^{-1}\end{array}\right)$, we may assume that $a=\operatorname{id}_{H}$ and $d=\mathrm{id}_{K}$. Now consider $g=\left(\begin{array}{cc}\operatorname{id}_{H} & b S \\ c S & \operatorname{id}_{K}\end{array}\right)$, another Hopf endomorphism of $H \otimes K$. One computes $g f=\left(\begin{array}{cc}\mathrm{id} * b c S & b * b S \\ c S * c & c b S * \mathrm{id}\end{array}\right)=\left(\begin{array}{cc}\mathrm{id} * b c S & \eta \varepsilon \\ \eta \varepsilon & c b S * \mathrm{id}\end{array}\right)$. By the chain conditions on $H$ and $K$, for $n$ sufficiently large $b$ and $c$ induce mutually inverse isomorphisms between the images of $(b c)^{n}$ and $(c b)^{n}$. Fitting's lemma implies that these isomorphic images are an abelian common tensor factor of $H$ and $K$. It can only be trivial if $b c$ and $c b$ are nilpotent, in which case id $* b c S$ and $c b S *$ id are automorphisms. In the latter case, $f$ was an automorphism.

These results have obvious extensions to more than two factors by induction, which we leave to the reader. The results, however, do not cover the case of a repeated tensor factor. For a given Hopf algebra $H$ in $\mathcal{B}$ we can form the unbraided iterated tensor product $H^{\otimes n}=H \otimes \cdots \otimes H$ for $n \in \mathbb{N}$ precisely when $H$ is in a (sub)category where the braiding is a symmetry.

Theorem 8.2. Let $H$ be a tensor indecomposable nonabelian Hopf algebra satisfying both chain conditions in $\mathcal{B}$, and suppose the braiding of $\mathcal{B}$ is a symmetry. Fix $n \in \mathbb{N}$, and let $\mathcal{A}_{n}$ denote those $\left(\alpha_{i j}\right) \in \operatorname{End}\left(H^{\otimes n}\right)$ such that $\alpha_{i i} \in \operatorname{Aut}(H)$ and $\alpha_{i j} \in \operatorname{End}^{c}(H)$ for all $i$ and $j \neq i$. Then

$$
\operatorname{Aut}\left(H^{\otimes n}\right) \cong \mathcal{A}_{n} \rtimes S_{n} .
$$

Proof. By assumptions on $H, \mathcal{A}_{n} \subseteq \operatorname{Aut}\left(H^{\otimes n}\right)$. The group $S_{n}$ acts on $H^{\otimes n}$ by permuting factors, and so acts on $\operatorname{Aut}\left(H^{\otimes n}\right)$ by permuting columns. Conjugating by this action sends $\mathcal{A}_{n}$ to itself. We need only show that every automorphism is a column permutation of an element of $\mathcal{A}_{n}$.

So let $\left(\alpha_{i j}\right) \in \operatorname{Aut}\left(H^{\otimes n}\right)$, with inverse $\left(\alpha_{i j}^{\prime}\right)$. Then $\alpha_{i 1} \alpha_{1 i}^{\prime} * \cdots * \alpha_{i n} \alpha_{n i}^{\prime}=\mathrm{id}$ holds for all $i$. Since the $\alpha_{i k} \alpha_{k i}^{\prime}$ are all binormal endomorphisms the notation is unambiguous, and the terms of the convolution product can be arbitrarily reordered. Moreover, since $H$ is indecomposable we may conclude that one of the $\alpha_{i k} \alpha_{k i}^{\prime}$ is an automorphism. In particular for all $i$ there is a $k$ such that $\alpha_{i k}$ is an epimorphism and $\alpha_{k i}^{\prime}$ is a monomorphism. By the chain conditions it follows that $\alpha_{i k}$ and $\alpha_{k i}^{\prime}$ are both automorphisms. Since $H$ is nonabelian there is at most one such $k$ for any given $i$. This completes the proof.

\section{Application to doubles of groups}

For this section we work in the category of vector spaces over a field $\mathbb{k}$. Throughout this section $G, H, K, C$ will all be finite groups. For any group $G$ let $\widehat{G}$ be the group of group-like elements of $\mathbb{k}^{G}$, the dual of the group algebra $\mathbb{k} G$. Note that $\widehat{G}$ is precisely the k-linear characters of $G$. We also define $\Gamma_{G}=\widehat{G} \times G$. We denote 
the conjugation action of $G$ on $\mathbb{k}^{G}$ and $\mathbb{k} G$ both by $\rightarrow$; for instance: $g \rightarrow x=g x g^{-1}$ for all $g, x \in G$. We will be concerned with $\mathcal{D}(G)$, the Drinfeld double of a finite group $G$. As a coalgebra $\mathcal{D}(G)=\mathbb{k}^{G c o} \otimes \mathbb{k} G$, and the algebra structure is given by having $G$ act on $\mathbb{k}^{G \text { co }}$ by the conjugation action. We note that $\Gamma_{G}$ is the group of group-like elements of $\mathcal{D}(G)$. See [Dijkgraaf et al. 1991; Montgomery 1993] for further details on the construction and properties of this Hopf algebra.

In [Keilberg 2015] the first author gave a complete description of $\operatorname{Aut}(\mathcal{D}(G))$ whenever $G$ has no nontrivial abelian direct factors. Such a group is said to be purely nonabelian. When $G$ is abelian we have $\mathcal{D}(G)=\mathbb{k}^{G} \otimes \mathbb{k} G$, an abelian Hopf algebra, and the determination of $\operatorname{Aut}(\mathcal{D}(G))$ is then straightforward. Indeed, under mild assumptions on $\mathbb{k}$ we have $\mathcal{D}(G) \cong \mathbb{k}(G \times G)$. Subsequently in this case $\operatorname{Aut}(\mathcal{D}(G))$ can be computed by classical methods in group theory [Shoda 1928]. We note that the structure of such an automorphism group has been of more recent interest [Bidwell and Curran 2010; Hillar and Rhea 2007]. It is the goal of this section to complete the description of $\operatorname{Aut}(\mathcal{D}(G))$ when $G$ has an abelian direct factor.

So suppose that $G=C \times H$ with $C$ abelian. Then $\mathcal{D}(G) \cong \mathcal{D}(C) \otimes \mathcal{D}(H)$. Since $\mathcal{D}(C)$ is an abelian Hopf algebra the results of the previous section can be applied whenever $\mathcal{D}(H)$ has no abelian direct tensor factors. We will proceed to show this happens precisely when $H$ is purely nonabelian.

We have the following description of $\operatorname{Hom}(\mathcal{D}(G), \mathcal{D}(K))$.

Theorem 9.1 [Keilberg 2015; Agore et al. 2014]. The elements of the set $\operatorname{Hom}(\mathcal{D}(G), \mathcal{D}(K))$ are in bijective correspondence with matrices $\left(\begin{array}{ll}u & r \\ p & v\end{array}\right)$ where $u: \mathbb{k}^{G} \rightarrow \mathbb{k}^{K}$ is a morphism of unitary coalgebras, $p: \mathbb{k}^{G} \rightarrow \mathbb{k} K$ is a morphism of Hopf algebras, and $r: G \rightarrow \widehat{K}$ and $v: G \rightarrow K$ are group homomorphisms. These are all subject to the following compatibility relations, for all $a, b \in \mathbb{k}^{G}$ and $g \in G$ :

(i) $u(g \rightarrow a)=v(g) \rightarrow u(a)$, from which it follows that $u^{*} v$ is normal;

(ii) $u \curlywedge p$;

(iii) $u(a b)=u\left(a_{(1)}\right)\left(p\left(a_{(2)}\right) \rightarrow u(b)\right)$;

(iv) $p(g \rightarrow a)=v(g) \rightarrow p(a)$.

The morphism is defined by

$$
a \# g \mapsto u\left(a_{(1)}\right) r(g) \# p\left(a_{(2)}\right) v(g) .
$$

Composition of such morphisms is given by matrix multiplication, as in Section 7.

The morphism $p$ is uniquely determined by an isomorphism $\mathbb{k}^{A} \cong \mathbb{k} B$, where $A$ is an abelian normal subgroup of $G$ and $B$ is an abelian subgroup of $K$. In particular we must have $\mathbb{k}^{A} \cong \mathbb{k} \widehat{A}$. For the remainder of this section any use of $A, B$ refers to these subgroups. We note that the last relation says $p \curlyvee v$ if and only if $A \leq Z(G)$, or equivalently $p$ is cocentral: $p$ 人 id. 
By convention we implicitly identify any element of $\operatorname{Hom}(\mathcal{D}(G), \mathcal{D}(K))$ or $\operatorname{Hom}\left(\mathbb{K}^{G} \otimes \mathbb{k} G, \mathbb{k}^{K} \otimes \mathbb{k} K\right)$ with its quadruple of components $(u, r, p, v)$, or equivalently as a matrix $\left(\begin{array}{ll}u & r \\ p & v\end{array}\right)$.

The following is then immediate.

Lemma 9.2. A morphism $\psi \in \operatorname{Hom}(\mathcal{D}(G), \mathcal{D}(K))$ is canonically an element of $\operatorname{Hom}\left(\mathbb{k}^{G} \otimes \mathbb{k} G, \mathbb{k}^{K} \otimes \mathbb{k} K\right)$ precisely when $p \curlyvee v$ and $u$ is a morphism of Hopf algebras. On the other hand, $\phi \in \operatorname{Hom}\left(\mathbb{k}^{G} \otimes \mathbb{k} G, \mathbb{k}^{K} \otimes \mathbb{k} K\right)$ is canonically an element of $\operatorname{Hom}(D(G), \mathcal{D}(K))$ precisely when $u^{*} v$ is normal and $A \leq Z(G)$.

In the first case we call such a morphism untwistable, and in the second we call it twistable. Clearly any untwistable morphism is also twistable, and vice versa. The distinction is simply in the algebra structures we start with.

Now since $\mathbb{k}^{G} \otimes \mathbb{k} G$ and $\mathbb{k}^{K} \otimes \mathbb{k} K$ are canonically self-dual, any morphism $\psi \in$ $\operatorname{Hom}\left(\mathbb{k}^{G} \otimes \mathbb{k} G, \mathbb{k}^{K} \otimes \mathbb{k} K\right)$ yields a dual morphism $\psi^{*} \in \operatorname{Hom}\left(\mathbb{k}^{K} \otimes \mathbb{k} K, \mathbb{k}^{G} \otimes \mathbb{k} G\right)$ with components $\left(v^{*}, r^{*}, p^{*}, u^{*}\right)$. The following is then clear.

Corollary 9.3. Both $\psi \in \operatorname{Hom}\left(\mathbb{k}^{G} \otimes \mathbb{k} G, \mathbb{k}^{K} \otimes \mathbb{k} K\right)$ and $\psi^{*}$ are twistable if and only if the following all hold:

(i) $u^{*} v$ is normal;

(ii) $v u^{*}$ is normal;

(iii) $A \leq Z(G)$;

(iv) $B \leq Z(K)$.

In this case we may canonically view $\psi$ as an element of $\operatorname{Hom}(\mathcal{D}(G), \mathcal{D}(K))$ and $\psi^{*}$ as an element of $\operatorname{Hom}(\mathcal{D}(K), \mathcal{D}(G))$.

In [Keilberg 2015] a morphism $\psi=(u, r, p, v) \in \operatorname{Hom}(\mathcal{D}(G), \mathcal{D}(K))$ was said to be flippable if also $\left(v^{*}, r^{*}, p^{*}, u^{*}\right) \in \operatorname{Hom}(\mathcal{D}(K), \mathcal{D}(G))$. This is equivalent to saying that $\psi$ is untwistable and the corresponding dual $\psi^{*}$ is twistable. In particular the corollary gives a complete description of the flippable elements of $\operatorname{Hom}(\mathcal{D}(G), \mathcal{D}(K))$, and shows that "flipping" an element of $\operatorname{Hom}(\mathcal{D}(G), \mathcal{D}(K))$ can naturally be described as dualizing the morphism.

Corollary 9.4. For any group $G$, the group $\operatorname{Aut}(\mathcal{D}(G))$ is canonically a subgroup of $\operatorname{Aut}\left(\mathbb{K}^{G} \otimes \mathbb{k} G\right)$ which is closed under dualization.

Proof. Follows from the preceding corollary, Section 8, and the properties of $\operatorname{Aut}(\mathcal{D}(G))$ established in [Keilberg 2015].

We now show that the act of untwisting a morphism is fairly well behaved whenever the image is commutative.

Proposition 9.5. Let $\psi \in \operatorname{Hom}(\mathcal{D}(G), \mathcal{D}(K))$ be untwistable. For convenience, let $\psi^{\prime}=\psi \in \operatorname{Hom}\left(\mathbb{k}^{G} \otimes \mathbb{k} G, \mathbb{k}^{H} \otimes \mathbb{k} H\right)$. Then the following all hold. 
(i) If $G=H$, then $\psi$ is conormal if and only if $\psi^{\prime}$ is conormal.

(ii) If $\psi$ has a commutative image then $\psi^{\prime}$ has commutative image.

(iii) If $\psi$ has commutative image and $G=H$, then $\psi^{\prime}$ is normal if and only if $v$ is normal and $B \leq Z(G)$.

(iv) If $\psi$ has commutative image and $G=H$, then $\psi$ is normal if and only if $\psi^{\prime}$ is normal and $G$ acts trivially on $\operatorname{Img}(u)$.

(v) If $\psi$ has commutative image and $G=H$, then $\psi$ is conormal.

Proof. We first prove (i), as it is the only one that does not suppose that $\psi$ has commutative image. To this end we compute

$$
\begin{aligned}
a_{(3)} \# g \cdot S\left(a_{(1)} \# g\right) \otimes a_{(2)} \# g & =a_{(3)} \# g \cdot\left(g^{-1} \rightarrow S\left(a_{(1)}\right) \# g^{-1}\right) \otimes a_{(2)} \# g \\
& =a_{(3)} S\left(a_{(1)}\right) \# 1 \otimes a_{(2)} \# g \\
a_{(3)} \otimes g \cdot S\left(a_{(1)} \otimes g\right) \otimes a_{(2)} \otimes g & =a_{(3)} S\left(a_{(1)}\right) \otimes 1 \otimes a_{(2)} \otimes g .
\end{aligned}
$$

The claim then follows.

For the remainder of the proof, suppose that $\psi$ is untwistable and has commutative image. By checking the commutativity condition we can easily determine the following facts: $p \curlyvee v$; $v$ has abelian image, or equivalently $v \curlyvee v ; u(a(g \rightarrow b))=$ $u((h \rightarrow a) b)=u(a b)$ for all $g, h \in G$ and $a, b \in \mathbb{k}^{G}$, which implies $u \curlywedge$ id. In particular, $u(g \rightarrow a)=u(a)$ and $p(g \rightarrow a)=p(a)$ for all such $a, g$.

The first part is then immediate, as we have $\psi(a \# g \cdot b \# h)=\psi^{\prime}(a \otimes g \cdot b \otimes h)$. Another way of saying this is that when $\psi$ has commutative image we may compute products in either the double or the tensor product without affecting the result. Furthermore $\psi((g \rightarrow a) \# g)=\psi(a \# g)$ for all appropriate $a, g$, and so

$$
\psi(S(a \# g))=\psi\left(g^{-1} \rightarrow S(a) \# g^{-1}\right)=\psi\left(S(a) \# g^{-1}\right)=\psi^{\prime}(S(a \otimes g)) .
$$

Thus we may perform all computations with $\psi$ in either $\mathcal{D}(G)$ or $\mathbb{k}^{G} \otimes \mathbb{k}_{k}$ as we desire.

The last part of the result follows from Equations (9-1) and (9-2) and $u$ 人 id. We need only prove the parts concerning normality of $\psi, \psi^{\prime}$.

To determine when $\psi^{\prime}$ is normal, we first note that by commutativity we have

$$
\psi^{\prime}\left(a_{(2)} \otimes g \cdot b \otimes h \cdot S\left(a_{(1)}\right) \otimes g^{-1}\right)=a(1) \psi^{\prime}(b \otimes h)
$$

On the other hand,

$$
a_{(2)} \otimes g \cdot \psi^{\prime}(b \otimes h) \cdot S\left(a_{(1)}\right) \otimes g^{-1}=a(1) r(h) u\left(b_{(1)}\right) \otimes g p\left(b_{(1)}\right) v(h) g^{-1} .
$$

The map $\psi^{\prime}$ is normal precisely when these two expressions are the same, and we easily find this is equivalent to $B \leq Z(H)$ and $v$ normal. 
Finally, we determine when $\psi$ is normal. By previous remarks, we have

$$
\begin{aligned}
\psi\left(a_{(2)} \# g \cdot b \# h \cdot S\left(a_{(1)} \# g\right)\right) & =a(1) \psi\left(b \# g h g^{-1}\right) \\
& =a(1) r(h) u\left(b_{(1)}\right) \# p\left(b_{(2)}\right) v\left(g h g^{-1}\right) .
\end{aligned}
$$

On the other hand, we have

$$
\begin{aligned}
a_{(2)} \# g \cdot \psi(b \# h) \cdot\left(g^{-1} \rightarrow S a_{(1)} \# g^{-1}\right) \\
\quad=r(h) a_{(2)}\left(g \rightarrow u\left(b_{(1)}\right)\right)\left(g p\left(b_{(2)}\right) v(h) g^{-1} \rightarrow S\left(a_{(1)}\right)\right) \# g p\left(b_{(3)}\right) v(h) g^{-1} .
\end{aligned}
$$

Applying $\varepsilon$ \# id to both expressions we get

$$
a(1) p(b) v\left(g h g^{-1}\right)
$$

for the first and

$$
a(1) g p(b) v(h) g^{-1}
$$

for the second. These are equal for all $a, b, g, h$ if and only if $B \leq Z(H)$ and $v$ is normal; equivalently, $\psi^{\prime}$ is normal. Note that if $v$ is normal and has abelian image, then its image is in fact central. Therefore $g p(b) v(h) g^{-1} \rightarrow S(a)=S(a)$ precisely when $\psi^{\prime}$ is normal. Subsequently the previous equation simplifies to

$$
a(1) r(h)\left(g \rightarrow u\left(b_{(1)}\right)\right) \# p\left(b_{(2)}\right) v\left(g h g^{-1}\right) .
$$

Comparing with (9-3) completes the proof.

Lemma 9.6. Any commutative direct tensor factor of $\mathcal{D}(G)$ is also a commutative direct tensor factor of $\mathbb{k}^{G} \otimes \mathbb{k} G$.

Proof. Suppose $L$ is a commutative Hopf subalgebra of $\mathcal{D}(G)$ such that $\mathcal{D}(G)=$ $M \otimes L$ for some Hopf subalgebra $M$. We then have a projection $\pi: \mathcal{D}(G) \rightarrow L$ with associated right inverse the imbedding $i: L \hookrightarrow \mathcal{D}(G)$.

The morphism $i \pi$ is an endomorphism of $\mathcal{D}(G)$. Since the image is central in $\mathcal{D}(G)$ it is easily seen to be untwistable and binormal. Therefore $i \pi$ is canonically a twistable, binormal, idempotent endomorphism of $\mathbb{k}^{G} \otimes \mathbb{k} G$ with image $L$. By Fitting's lemma we conclude that $L$ is also a direct tensor factor of $\mathbb{k}^{G} \otimes \mathbb{k} G$.

Remark 9.7. Since $\mathbb{k}^{G}$ is commutative we see that the converse will only hold when $G$ is abelian. Indeed since $\mathcal{D}(G)$ is quasitriangular any commutative direct tensor factor of $\mathcal{D}(G)$ is necessarily abelian.

The lemma gives one part of the following. 
Theorem 9.8. Let $G$ be a finite group. Then the following are equivalent.

(i) $G$ is purely nonabelian.

(ii) $\mathbb{k} G$ is purely nonabelian.

(iii) $\mathbb{k}^{G}$ is purely nonabelian.

(iv) $\mathbb{k}^{G} \otimes \mathbb{k} G$ is purely nonabelian.

(v) $\mathcal{D}(G)$ is purely nonabelian.

Indeed, $\mathbb{k}^{G} \otimes \mathbb{k} G$ and $\mathcal{D}(G)$ have the same abelian direct tensor factors.

Proof. Since the dual of an abelian Hopf algebra is again abelian, the equivalence of the second and third is immediate. By Krull-Remak-Schmidt, any abelian indecomposable factor of $\mathbb{k}^{G} \otimes \mathbb{k} G$ is isomorphic to an abelian indecomposable factor of either $\mathbb{k} G$ or $\mathbb{k}^{G}$. Thus the fourth is equivalent to the second and third. Since any Hopf subalgebra of $\mathbb{k} G$ is a subgroup algebra, the first and second are equivalent. By the lemma the fourth implies the fifth. To prove the fifth implies the fourth, we need only show that any abelian factor of $\mathbb{k}^{G} \otimes \mathbb{k} G$ yields an abelian factor of $\mathcal{D}(G)$.

So let $L$ be an abelian tensor factor of $\mathbb{k}^{G} \otimes \mathbb{k} G$ with associated projection $\pi$ and inclusion $i$. We wish to show that $i \pi$ is canonically a binormal endomorphism of $\mathcal{D}(G)$. Writing $i \pi=\left(\begin{array}{ll}u & r \\ p & v\end{array}\right)$, the properties of $\operatorname{End}(\mathcal{D}(G))$ and commutativity of the image easily imply the following: $p \curlyvee v, v \curlyvee v, u \curlywedge p, p \curlyvee$ id, $v \curlyvee$ id. In particular, $v$ and $p$ have central image, and $v$ is a (bi)normal group homomorphism. Since $(i \pi)^{*}$ is also an idempotent endomorphism with abelian image we similarly conclude that $p^{*}$ and $u^{*}$ have central image, and that $u^{*}$ is a (bi)normal group homomorphism. Centrality of the image of $u^{*}$ (indeed, that $u^{*}$ has abelian image and is thus a class function) implies that $G$ acts trivially on the image of $u$. Applying the proposition we conclude that $i \pi$ is canonically a binormal endomorphism of $\mathcal{D}(G)$ with image $L$. Fitting's lemma then implies that $L$ is a direct tensor factor of $\mathcal{D}(G)$, as desired. This completes the proof.

Thus for $G=C \times H$ with $C$ abelian and $H$ purely nonabelian we conclude that $\mathcal{D}(C)$ and $\mathcal{D}(H)$ have no common direct tensor factors. Therefore we may apply the results of the previous section to obtain the following.

Theorem 9.9. Let $G=C \times H$, where $C, H$ are finite groups with $C$ abelian and $H$ purely nonabelian. Then

$$
\operatorname{Aut}(\mathcal{D}(G))=\left(\begin{array}{cc}
\operatorname{Aut}(\mathcal{D}(C)) & \operatorname{Hom}^{c}(\mathcal{D}(H), \mathcal{D}(C)) \\
\operatorname{Hom}^{c}(\mathcal{D}(C), \mathcal{D}(H)) & \operatorname{Aut}(\mathcal{D}(H))
\end{array}\right) .
$$

The determination of the $\mathrm{Hom}^{c}$ terms remains a computational problem, but all of the components of these morphisms are guaranteed to be morphisms of Hopf algebras, and so determined by group homomorphisms. We note that for 
$\operatorname{Hom}^{c}(\mathcal{D}(H), \mathcal{D}(C))$ we have a commutative image, as considered in Proposition 9.5. Whenever the field is such that $\mathcal{D}(C)$ is just a group algebra then the situation is further simplified. In this case $\operatorname{Hom}^{c}(\mathcal{D}(C), \mathcal{D}(H))=\operatorname{Hom}\left(\Gamma_{C}, Z\left(\Gamma_{H}\right)\right)$, a group of morphisms between abelian groups. Furthermore, it is straightforward to check that $\left(\begin{array}{ll}u & r \\ p & v\end{array}\right) \in \operatorname{Hom}(\mathcal{D}(H), \mathcal{D}(C))$ defines an element of $\operatorname{Hom}^{c}(\mathcal{D}(H), \mathcal{D}(C))$ if and only if $u \curlywedge$ id and $p \curlywedge$ id; note that $p \curlywedge$ id if and only if $A \leq Z(H)$.

Example 9.10. Consider a field $\mathbb{k}$ of characteristic not 2. For $n \geq 3$, let $G=D_{2 n}$ be the dihedral group of order $2 n$, and suppose that $n \equiv 2 \bmod 4$. The group $G$ has an abelian direct factor precisely under this assumption on $n$, in which case $G \cong \mathbb{Z}_{2} \times D_{n}$. So we take $C=\mathbb{Z}_{2}$ and $H=D_{n}$, and note $\Gamma_{C} \cong \mathbb{Z}_{2}^{2}$ and $\Gamma_{H} \cong \mathbb{Z}_{2} \times D_{n}$. It is also well known that $\operatorname{Aut}\left(\Gamma_{C}\right) \cong S_{3}$. By [Keilberg 2015] we have $\operatorname{Aut}\left(\mathcal{D}\left(D_{n}\right)\right) \cong \mathbb{Z}_{2} \times \operatorname{Aut}\left(D_{n}\right) \cong \mathbb{Z}_{2} \times \operatorname{Hol}\left(\mathbb{Z}_{n / 2}\right)$. Here $\operatorname{Hol}\left(\mathbb{Z}_{n}\right)=\mathbb{Z}_{n} \rtimes \operatorname{Aut}\left(\mathbb{Z}_{n}\right)$ is the holomorph of $\mathbb{Z}_{n}$, a group of order $n \phi(n)$, where $\phi$ is the Euler totient function.

We have $Z\left(\Gamma_{H}\right) \cong \mathbb{Z}_{2}$, from which it follows that $\operatorname{Hom}\left(\Gamma_{C}, Z\left(\Gamma_{H}\right)\right) \cong \mathbb{Z}_{2}^{2}$ as groups. We claim that

$$
\operatorname{Hom}^{c}(\mathcal{D}(H), \mathcal{D}(C)) \cong \mathbb{Z}_{2}^{2}
$$

as well. Let $(u, r, p, v) \in \operatorname{Hom}(\mathcal{D}(H), \mathcal{D}(C))$. The abelian normal subgroups of $D_{n}$ all have odd order, so $p$ is necessarily trivial. By normality of $u^{*} v$, we have $u^{*}\left(b^{v(x)}\right)=u^{*}(b)=u^{*}(b)^{x}$ for all $x \in D_{n}$ and $b \in \mathbb{Z}_{2}$. Since no order 2 subgroup of $D_{n}$ is normal we conclude that $u^{*}$ is trivial. From the preceding remarks it follows that $\operatorname{Hom}(\mathcal{D}(H), \mathcal{D}(C))=\operatorname{Hom}^{c}(\mathcal{D}(H), \mathcal{D}(C))$. Since there are two possible homomorphisms $v: D_{n} \rightarrow \mathbb{Z}_{2}$, and two possible homomorphisms $r: D_{n} \rightarrow \widehat{\mathbb{Z}}_{2}$, all of which satisfy the necessary compatibilities, it quickly follows that $\operatorname{Hom}^{c}(\mathcal{D}(H), \mathcal{D}(C)) \cong \mathbb{Z}_{2}^{2}$ as desired.

As a consequence, $\left|\operatorname{Aut}\left(\mathcal{D}\left(D_{2 n}\right)\right)\right|=2^{5} \cdot 3 \cdot n \cdot \phi(n / 2)$ whenever $n \equiv 2 \bmod 4$. For $n=6$ the order is $1152=2^{7} \cdot 3^{2}$. The description and order of $\operatorname{Aut}\left(\mathcal{D}\left(D_{2 n}\right)\right)$ for $n \not \equiv 2 \bmod 4$ is given in [Keilberg 2015].

\section{References}

[Agore et al. 2014] A. L. Agore, C. G. Bontea, and G. Militaru, "Classifying bicrossed products of Hopf algebras”, Algebr. Represent. Theory 17:1 (2014), 227-264. MR 3160722 Zbl 06306463

[Bespalov and Drabant 1998] Y. Bespalov and B. Drabant, "Hopf (bi-)modules and crossed modules in braided monoidal categories", J. Pure Appl. Algebra 123:1-3 (1998), 105-129. MR 99f:18012 Zbl 0902.16029

[Bidwell 2008] J. N. S. Bidwell, "Automorphisms of direct products of finite groups II", Arch. Math. (Basel) 91:2 (2008), 111-121. MR 2009f:20030 Zbl 1185.20023

[Bidwell and Curran 2010] J. N. S. Bidwell and M. J. Curran, "Automorphisms of finite abelian groups", Math. Proc. R. Ir. Acad. 110A:1 (2010), 57-71. MR 2011g:20087 Zbl 1275.20018 
[Bidwell et al. 2006] J. N. S. Bidwell, M. J. Curran, and D. J. McCaughan, "Automorphisms of direct products of finite groups", Arch. Math. (Basel) 86:6 (2006), 481-489. MR 2007e:20047 Zbl 1103.20016

[Burciu 2011] S. Burciu, "On complements and the factorization problem of Hopf algebras", Cent. Eur. J. Math. 9:4 (2011), 905-914. MR 2012d:16095 Zbl 1263.16031

[Chirvăsitu 2010] A. Chirvăsitu, "On epimorphisms and monomorphisms of Hopf algebras", $J$. Algebra 323:5 (2010), 1593-1606. MR 2011f:16075 Zbl 1198.16030

[Dijkgraaf et al. 1991] R. Dijkgraaf, V. Pasquier, and P. Roche, "Quasi Hopf algebras, group cohomology and orbifold models", pp. 60-72 in Recent advances in field theory (Annecy-le-Vieux, 1990), vol. 18B, edited by P. Binétruy et al., Elsevier, Amsterdam, 1991. Nuclear Phys. B Proc. Suppl. MR 92m:81238 Zbl 0957.81670

[Hillar and Rhea 2007] C. J. Hillar and D. L. Rhea, "Automorphisms of finite abelian groups", Amer. Math. Monthly 114:10 (2007), 917-923. MR 2363058 Zbl 1156.20046

[Keilberg 2015] M. Keilberg, "Automorphisms of the doubles of purely non-abelian finite groups", Algebr. Represent. Theory 18:5 (2015), 1267-1297. MR 3422470

[Montgomery 1993] S. Montgomery, Hopf algebras and their actions on rings, CBMS Regional Conference Series in Mathematics 82, Amer. Math. Soc., Providence, RI, 1993. MR 94i:16019 Zbl 0793.16029

[Müger 2003] M. Müger, "On the structure of modular categories", Proc. London Math. Soc. (3) 87:2 (2003), 291-308. MR 2004g:18009 Zbl 1037.18005

[Nichols and Zoeller 1989] W. D. Nichols and M. B. Zoeller, "A Hopf algebra freeness theorem", Amer. J. Math. 111:2 (1989), 381-385. MR 90c:16008 Zbl 0672.16006

[Radford 1985] D. E. Radford, “The structure of Hopf algebras with a projection”, J. Algebra 92:2 (1985), 322-347. MR 86k:16004 Zbl 0549.16003

[Remak 1911] R. Remak, "Über die Zerlegung der endlichen Gruppen in direkte unzerlegbare Faktoren”, J. Reine Angew. Math. 139 (1911), 293-308. MR 1580820 JFM 48.1347.01

[Rotman 1995] J. J. Rotman, An introduction to the theory of groups, 4th ed., Graduate Texts in Mathematics 148, Springer, New York, 1995. MR 95m:20001 Zbl 0810.20001

[Scharfschwerdt 2001] B. Scharfschwerdt, "The Nichols Zoeller theorem for Hopf algebras in the category of Yetter Drinfeld modules”, Comm. Algebra 29:6 (2001), 2481-2487. MR 2002e:16064 Zbl 0984.16037

[Schauenburg 1998] P. Schauenburg, "On the braiding on a Hopf algebra in a braided category", New York J. Math. 4 (1998), 259-263. MR 99j:18008 Zbl 0914.16017

[Shoda 1928] K. Shoda, "Über die Automorphismen einer endlichen Abelschen Gruppe", Math. Ann. 100:1 (1928), 674-686. MR 1512510 JFM 56.0130.01

Communicated by Susan Montgomery

Received 2014-10-22 Accepted 2015-09-09

keilberg@usc.edu Institut de Mathématiques de Bourgogne, UMR5584 CNRS, Université Bourgogne Franche-Comté, F-21000 Dijon, France

peter.schauenburg@u-bourgogne.fr

Institut de Mathématiques de Bourgogne, UMR5584 CNRS, Université Bourgogne Franche-Comté, F-21000 Dijon, France 


\section{Algebra \& Number Theory}

msp.org/ant

\section{EDITORS}

MANAGING EDITOR

Bjorn Poonen

Massachusetts Institute of Technology

Cambridge, USA

\author{
EDITORIAL BOARD CHAIR \\ David Eisenbud \\ University of California \\ Berkeley, USA
}

BOARD OF EDITORS

Georgia Benkart

Dave Benson

Richard E. Borcherds

John H. Coates

J-L. Colliot-Thélène

Brian D. Conrad

Hélène Esnault

Hubert Flenner

Sergey Fomin

Edward Frenkel

Andrew Granville

Joseph Gubeladze

Roger Heath-Brown

Craig Huneke

Kiran S. Kedlaya

János Kollár

Yuri Manin

Philippe Michel
University of Wisconsin, Madison, USA

University of Aberdeen, Scotland

University of California, Berkeley, USA

University of Cambridge, UK

CNRS, Université Paris-Sud, France

Stanford University, USA

Freie Universität Berlin, Germany

Ruhr-Universität, Germany

University of Michigan, USA

University of California, Berkeley, USA

Université de Montréal, Canada

San Francisco State University, USA

Oxford University, UK

University of Virginia, USA

Univ. of California, San Diego, USA

Princeton University, USA

Northwestern University, USA

École Polytechnique Fédérale de Lausanne
Susan Montgomery

Shigefumi Mori

Raman Parimala

Jonathan Pila

Anand Pillay

Victor Reiner

Peter Sarnak

Joseph H. Silverman

Michael Singer

Vasudevan Srinivas

J. Toby Stafford

Ravi Vakil

Michel van den Bergh

Marie-France Vignéras

Kei-Ichi Watanabe

Efim Zelmanov

Shou-Wu Zhang
University of Southern California, USA

RIMS, Kyoto University, Japan

Emory University, USA

University of Oxford, UK

University of Notre Dame, USA

University of Minnesota, USA

Princeton University, USA

Brown University, USA

North Carolina State University, USA

Tata Inst. of Fund. Research, India

University of Michigan, USA

Stanford University, USA

Hasselt University, Belgium

Université Paris VII, France

Nihon University, Japan

University of California, San Diego, USA

Princeton University, USA

\section{PRODUCTION}

production@msp.org

Silvio Levy, Scientific Editor

See inside back cover or msp.org/ant for submission instructions.

The subscription price for 2016 is US $\$ 290 /$ year for the electronic version, and $\$ 485 /$ year ( $+\$ 55$, if shipping outside the US) for print and electronic. Subscriptions, requests for back issues and changes of subscribers address should be sent to MSP.

Algebra \& Number Theory (ISSN 1944-7833 electronic, 1937-0652 printed) at Mathematical Sciences Publishers, 798 Evans Hall \#3840, c/o University of California, Berkeley, CA 94720-3840 is published continuously online. Periodical rate postage paid at Berkeley, CA 94704, and additional mailing offices.

ANT peer review and production are managed by EditFLOW ${ }^{\circledR}$ from MSP.

\section{PUBLISHED BY}

- mathematical sciences publishers

nonprofit scientific publishing

http://msp.org/

(C) 2016 Mathematical Sciences Publishers 


\section{Algebra \& Number Theory}

Volume $10 \quad$ No. $1 \quad 2016$

Stable sets of primes in number fields

ALEXANDER IVANOV

Hopf-Galois structures arising from groups with unique subgroup of order $p$

Tiмотну KонL

On tensor factorizations of Hopf algebras

Marc Keilberg and Peter Schauenburg

Extension theorems for reductive group schemes

ADRIAN VASIU

Actions of some pointed Hopf algebras on path algebras of quivers

RYAN Kinser and CHELSEA WALTON

On the image of the Galois representation associated to a non-CM Hida family

JACLYN LANG

Linear relations in families of powers of elliptic curves

FABRIZIO BARROERO and LAURA CAPUANO 